\title{
Necroptosis inhibition counteracts axonal degeneration, cognitive decline and key hallmarks of aging, promoting brain rejuvenation.
}

Macarena S. Arrázola ${ }^{1,2}$, Matías Lira ${ }^{3}$, Gabriel Quiroz², Somya lqbal ${ }^{4}$, Samantha L Eaton ${ }^{4}$, Rachel A Kline $^{4}$, Douglas J Lamont ${ }^{4}$, Hernán Huerta ${ }^{1,2}$, Gonzalo Ureta ${ }^{7}$, Sebastián Bernales ${ }^{7}$, J César Cárdenas ${ }^{1,2,6,8}$, Waldo Cerpa ${ }^{3,5}$, Thomas M. Wishart ${ }^{4}$ and Felipe A. Court 1,2,6,*.

${ }^{1}$ Center for Integrative Biology, Faculty of Sciences, Universidad Mayor

${ }^{2}$ Geroscience Center for Brain Health and Metabolism (GERO), Santiago, Chile.

${ }^{3}$ Departamento de Biología Celular y Molecular, Facultad de Ciencias Biológicas, Pontificia Universidad Católica de Chile, Av. Libertador Bernardo O’Higgins 340, Santiago, Chile.

4 The Roslin Institute, University of Edinburgh, Edinburgh, UK.

${ }^{5}$ Centro de Excelencia en Biomedicina de Magallanes (CEBIMA), Universidad de Magallanes, Punta Arenas, Chile.

${ }^{6}$ Buck Institute for Research on Aging, Novato, CA, USA.

${ }^{7}$ Fundación Ciencia \& Vida, Santiago, Chile.

${ }^{8}$ Department of Chemistry and Biochemistry, University of California, Santa Barbara, California, USA

*Contact information: felipe.court@umayor.cl 


\begin{abstract}
Age is the main risk factor for cognitive impairment and the development of neurodegenerative diseases. In the aged brain, axonal degeneration is an early pathological event, preceding neuronal dysfunction and brain disabilities in humans, primates, rodents, and invertebrates. Necroptosis activation mediates degeneration of mechanical and chemically injured axons, but whether this pathway triggers axonal degeneration and cognitive impairment during brain aging has not been studied. Here we show that necroptosis is activated in the hippocampus during aging, especially in axonal tracts. Loss of the main necroptotic effector, Mlkl, was sufficient to delay age-associated axonal degeneration. Accordingly, aged MIkl-KO mice also displayed a youthful phenotype at the synaptic and functional level, protecting against decreased synaptic transmission and memory decline. Short-term pharmacologic inhibition of necroptosis by targeting RIPK3 in aged mice, proved to be extraordinarily effective at reverting axonal degeneration and hippocampal-dependent functional impairment at the electrophysiological and behavioral level. Remarkably, a comprehensive quantitative proteomic analysis uncovered a set of aging hallmarks that were recovered in both, the genetic and pharmacologic models of necroptosis inhibition, including molecular biofunctions associated with brain rejuvenation. Taken together, these findings demonstrate that necroptosis contributes to the age-associated deterioration of axonal integrity, affecting hippocampal neuronal connectivity and cognitive function in aged individuals. We therefore propose necroptosis as an attractive target for the future development of geroprotective tools to treat age-related disabilities.
\end{abstract}




\section{INTRODUCTION}

During the last two centuries, global economic progress and public health policies have been focused on reducing mortality rates, thereby raising human life expectancy (Wyss-Coray 2016; Aburto et al. 2020). Unfortunately, this increase in lifespan is not accompanied by an equivalent increase in 'healthspan', the functional and disease-free period of life in the elderly (Hansen \& Kennedy 2016). The impact of aging on brain function is unquestionable. Age is the main risk factor for the development of neurodegenerative diseases and associated cognitive disabilities (Duan et al. 2020; Agüero-Torres et al. 2002; Wyss-Coray 2016). According to the World Health Organization, brain disorders associated with advancing age such as Alzheimer's disease and other dementias are in the top causes of death worldwide (World Health Organization 2019), and they have become the most dreaded maladies in older people.

As a fundamental structure for human cognition, the hippocampus is particularly vulnerable to the deleterious effects of aging (O'Shea et al. 2016; Toner et al. 2009; Wimmer et al. 2012). Agerelated neuronal loss has been suggested in the hippocampus (Fu et al. 2015), however its involvement with impaired cognition in aging is still controversial (West 1993; Rasmussen et al. 1996; Shamy et al. 2006). Microstructural changes at the level of synaptic connections better predict learning and memory impairment along with aging. Decreased number of axospinous synapses have been described in the dentate gyrus of aged hippocampus (Geinisman et al. 1992) in association with decreased synaptic protein levels in memory-impaired aged rats (Smith et al. 2000). Interestingly, the reduced number of synaptic contacts during aging is correlated with a decrease in the presynaptic fiber potential due to a reduction of axons (Barnes \& McNaughton 1980), which contributes to the impaired synaptic plasticity and cognitive deficits experienced by aged organisms (Rosenzweig \& Barnes 2003).

White matter abnormalities and axonal degeneration ( $\mathrm{AxD}$ ) have been identified in aged brains of diverse species, including humans, primates, mice and flies (Garde et al. 2000; Peters et al. 2000; Stahon et al. 2016; Hussain et al. 2018). Altered axonal integrity and fiber density loss, occurs in hippocampal subfields as a function of age in humans, and they are strictly correlated with impaired memory performance (Radhakrishnan et al. 2020). Due to the importance of axonal integrity on hippocampal function and the progression of the cognitive decline seen in the elderly (Marner et al. 2003; Smith et al. 2004), it is imperative to determine the mechanism by which axons degenerate during aging. We were pioneers in identifying necroptosis as the mechanism regulating mechanical and chemical-induced AxD (Arrázola et al. 2019; Arrazola \& Court 2019; Hernández et al. 2018). More recently, it has been shown in a model of inflammatory-mediated axonal degeneration, that necroptosis activates Sarm1, a central executioner of pathological AxD (Gerdts et al. 2013; Osterloh et al. 2012; Ko et al. 2020). Necroptosis is an alternative form of programmed cell death characterized by a necrotic-like and pro-inflammatory cellular response (Molnár et al. 2019; Seo et al. 2021). 
Necroptosis is triggered by the tumor necrosis factor of cytokines under caspase-8 inhibitory conditions (Holler et al. 2000). Upon activation, receptor-interacting kinase 1 (RIPK1) recruits and phosphorylates RIPK3 which in turn phosphorylates the pseudo-kinase called mixed lineage kinase domain-like protein (MLKL) (Sun et al. 2012). Upon RIPK3-dependent phosphorylation, MLKL oligomerizes and translocate from the cytosol to the plasma membrane, disrupting membrane integrity followed by the release of cellular components, an exacerbated inflammatory response, and cell death (Cai et al. 2014; Samson et al. 2020).

Age-associated increase in chronic, low-grade sterile inflammation is one of the 'seven pillars of aging' that contributes to the development and progression of age-associated diseases (Kennedy et al. 2014; Franceschi \& Campisi 2014). Considering the inflammatory response triggered upon necroptosis activation, the role of this regulated cell death pathway in the progression of normal aging has been largely overlooked. Recent studies have depicted the importance of necroptosis in the aging of the mouse male reproductive system ( $\mathrm{Li}$ et al. 2017) and the epididymal white adipose tissue (Deepa et al. 2018). In the context of brain aging, several age-related neurodegenerative conditions that share prominent $\mathrm{AxD}$ and neuroinflammation as common features, such as Amyotrophic lateral sclerosis (ALS), Parkinson's disease (PD), Alzheimer's disease (AD), and virus-mediated neuroinflammation, have shown increased necroptosis activation in the brain, associated with functional impairment (Re et al. 2014; Ito et al. 2016; lannielli et al. 2018; Oñate et al. 2020; Caccamo et al. 2017; Bian et al. 2017; Daniels et al. 2017). These antecedents highlight the participation of necroptosis in the pathogenesis of age-related disorders. However, the involvement of necroptosis in the progression of normal brain aging and its cognitive consequences remains unexplored.

Here, we investigated the role of necroptosis in the progression of $A x D$ in the hippocampus as an early hallmark of normal aging. Necroptosis activation, evaluated by MLKL phosphorylation, increased in hippocampal subfields with prominent $A x D$ in aged mice. Additionally, loss of MIkl was sufficient to delay age-related AxD. This youthful phenotype in the hippocampus of aged Mlkl-ko mice was also displayed at the synaptic and functional level. Electrophysiological and behavioral evaluations demonstrated restored synaptic transmission and facilitation, accompanied by improved learning and memory performances in aged mice deficient for MIkl. Pharmacologic inhibition of RIPK3 in aged mice demonstrated to be extraordinarily effective on reverting $A x D$ and hippocampusdependent functional impairment. Finally, using an unbiased quantitative proteomic analysis, we demonstrate that genetic and pharmacologic inhibition of necroptosis leads to an overall improvement of the aged hippocampal proteome, restoring the levels of key protein pathways associated with the hallmarks of aging, including a subset of biofunctions associated with brain rejuvenation. Our study demonstrates that necroptosis contributes to the age-associated deterioration of axonal integrity and function, affecting hippocampal neuronal connectivity, and consequently the cognitive function of aged individuals. Finally, our pharmacological approach demonstrated that short-term treatment with 
a RIPK3 inhibitor promotes brain rejuvenation in aged mice, implicating necroptosis as an interesting target for the future development of therapeutic tools to treat age-related brain disabilities.

\section{RESULTS}

\subsection{Brain aging is associated with axonal degeneration in the hippocampal dentate gyrus.}

In order to establish the progression of hippocampal neurodegeneration throughout aging we used three groups of mice of different ages: adult (3-6 months), old (12-15 months) and aged (more than 20 months). Neurodegeneration, evaluated by Fluoro Jade C (FJC) staining (Fig 1a), significantly increases in the dentate gyrus (DG) of the hippocampus of old mice, reaching a plateau in the aged group (Fig 1b). Interestingly, increased neurodegeneration was restricted to the DG hilus, where DG granular neurons extend their axons, known as mossy fibers, to connect with CA3 pyramidal neurons (Kassab \& Alexandre 2018). FJC staining was also analyzed in other brain regions, as the striatum, cerebellum and spinal cord, in which age-dependent neurodegeneration was also evident (Fig S1).

To further evaluate age-associated axonal degeneration in the hippocampus, we studied the expression of two phosphorylated forms of neurofilaments (NFs), the main proteins shaping the axonal cytoskeleton. Most NFs are highly phosphorylated under physiological conditions (Petzold 2005), while increased non-phosphorylated NF (non-pNF) immunoreactivity is associated with inflammation, neurodegenerative diseases and brain injury (Tsunoda \& Fujinami 2002; Seehusen \& Baumgärtner 2010; Bock et al. 2013; Nadeem et al. 2016). Interestingly, almost undetectable immunoreactivity of non-pNF was observed in the DG of adult mice, while a progressive increase was evidenced during aging (Fig 1c, d). Axonal degeneration was also evaluated using a pan-axonalNF antibody to detect stable axonal NFs (Petzold 2005; Yuan et al. 2012) (Fig 1e). The integrity index was used to quantify axonal stability as we previously described (Arrázola et al. 2019). Axonal integrity significantly decreases in old and aged hippocampal DG compared with adult mice (Fig 1f). Using different degenerative readouts, these results illustrate the progression of axonal degeneration in the hippocampus during aging.

\subsection{Necroptosis activation in hilar cells and axonal fibers in the dentate gyrus of the aged hippocampus.}

Previous studies have indicated that axons degenerate by an apoptosis-independent mechanism (Finn et al. 2000; Whitmore et al. 2003). We recently demonstrated that mechanical and chemical-induced axonal degeneration is regulated by necroptosis (Arrázola et al. 2019). To determine whether necroptosis is involved in age-associated axonal degeneration, we first assessed the activation of the necroptotic pathway by detecting the phosphorylated form of MLKL (pMLKL) in the hippocampus. The analysis was performed in the hilus and the granular cell layer (GCL) of the 
DG (Fig 2b). The number of pMLKL positive hilar cells increased in aged mice, as did PMLKL mean intensity (Fig 2a, c). Necroptosis activation was also evidenced by an age-dependent translocation of pMLKL from the nucleus to the cytoplasm (Fig 2a, magnified image), as has been previously described (Yoon et al. 2016; Weber et al. 2018). Non-significant changes in pMLKL levels were observed in GCL neurons (data not shown), suggesting a particular vulnerability of DG hilus to necroptosis activation during aging. Axonal pMLKL staining was evaluated in pan-axonal-NF positive hilar fibers of the DG (Fig 2d). Interestingly, pMLKL mean intensity increased earlier in DG axons compared with dentate hilar cells throughout aging, reaching significant differences from the old mice group (Fig 2e). Increased pMLK signal was also observed in other brain regions with defined axonal subfields, as axonal tracts in the striatum, the cerebellar white matter and the ventral horn of the spinal cord, which also showed progressive axonal degeneration along aging (Fig S2). The increase in MLKL phosphorylation levels in the hilus was also accompanied by changes in the pattern of pMLKL signal, from almost non-detected in adult mice axons, diffuse in the old group, to finally become punctuated in fragmented axons of aged mice (Fig 2d, magnified image). These aggregates of pMLKL have been associated with MLKL oligomerization and its translocation to the plasma membrane (Yoon et al. 2016), two key steps for necroptosis execution (Chen et al. 2014). A similar pattern of pMLKL was observed by immunohistochemistry against pMLKL in the aged hippocampus, both in DG hilar cells and in Schaffer collateral axons (Fig 2f), which connect with CA1 pyramidal neurons. Taken together, these results indicate that necroptosis is activated during aging in the hippocampus, particularly in the DG hilus, an hippocampal region recognized as one of the most vulnerable to the effects of aging (Spiegel et al. 2013).

\subsection{Age-induced axonal degeneration is reduced in MLKL knockout mice.}

Due to the indispensable role of MLKL in executing necroptosis, we evaluated whether agedependent axonal degeneration in the hippocampus was modified in aged MIkl-knockout mice (MIklKO) (Wu et al. 2013). Interestingly, axonal degeneration evaluated with a degeneration index (DI) in pan-axonal NF-stained axons was significantly reduced in aged MIkl-KO mice at levels comparable with adult mice (Fig 3a, b). Moreover, non-pNF degenerated axons profusely present in aged WT hippocampus were almost undetected in aged MIkl-KO mice, also at levels equivalent to younger WT mice (Fig 3a, c). As expected, we observed age-dependent axonal degeneration coupled with neuroinflammation (Hwang et al. 2018; von Bernhardi et al. 2015) (Fig S3). The increased number of microglia in the hippocampus of aged mice was not observed in aged Mlkl-KO mice (Fig S4). Accordingly, measurement of Iba1 mean intensity also indicated that aged MIkl-KO mice present less microglia activation than their WT littermates (Fig $\mathbf{3 d}$, e). To confirm the contribution of necroptosis in the inflammatory state of the brain, we measured the levels of several cytokines and chemokines in hippocampal lysates of adult versus aged WT and MIkl-KO mice by Luminex High Performance Assay (Table S1). Three of the twelve cytokines analyzed showed significant changes under MLKL deficiency in the hippocampus of aged mice. The levels of the pro-inflammatory cytokine IL-12 
decreased in aged MIkl-KO hippocampus compared with aged WT mice, reaching levels comparable with adult WT mice (Fig 3f). Interestingly, the anti-inflammatory cytokines IL-2 and IL-10 significantly increased in the hippocampus of aged Mlkl-KO mice (Fig 3f). Moreover, the systemic proinflammatory profile also decreased in serum samples from aged Mlkl-KO mice (Fig S5). These results indicate that necroptosis contributes to brain inflammation by modulating both pro- and antiinflammatory cytokines, but also controlling systemic inflammation.

\subsection{Loss of MLKL improves hippocampal synaptic transmission, learning and memory in aged mice.}

To further study whether necroptosis-mediated axonal degeneration affects hippocampal function along aging, we first performed electrophysiological recordings of the CA3-CA1 synapses to evaluate synaptic transmission. Extracellular field-excitatory post-synaptic potentials (fEPSP) were registered in hippocampal slices (Fig 4a). Average traces of the evoked potential recorded are represented in Fig $4 \mathbf{b}$ for each group. An age-dependent decrease in fEPSP slope was observed in WT mice. Nevertheless, in aged Mlkl-KO mice, the fEPSP slope was maintained at levels comparable to adult WT mice (Fig 4c, d). To specifically evaluate whether axonal alterations contribute to an agedependent decrease in synaptic transmission, we analyzed the facilitation index (fEPSP $2 / \mathrm{fEPSP}_{1}$ ) using a paired-pulse stimulation protocol (Fig 4e). The increased facilitation index observed in WT aged mice (Fig 4f) indicates a decreased neurotransmitter release probability from the axonal compartment (pre-synapse). Interestingly, the facilitation index of aged Mlkl-KO mice was comparable to adult WT mice (Fig 4e, f). These results indicate that Mlkl deficiency delays the loss of synaptic strength in the hippocampus inherent to brain aging, mainly preventing axonal function defects in aged mice.

Since memory capabilities depend on proper hippocampal function and both are affected by age (Yang et al. 2019; Burke \& Barnes 2006), we evaluated whether MIkl loss improves spatial learning and memory in aged mice. To this end, a Morris water maze (MWM) navigation task was performed in adult and aged MIkl -KO mice and compared with their WT littermates. Mice were trained to locate a hidden platform for 4 consecutive days. In this training scheme, the learning curve of aged MIkl-KO mice was significantly faster than those of aged WT animals and reached a reduced latency to find the platform from day 3 until the end of the training, showing comparable escape time to adult animals.

(Fig 4g). No differences were observed between adult WT and Mlkl-KO mice in the learning curve. To evaluate memory, 24 hours after training, mice were challenged to find the original location of the platform, which was removed. The differences between adult and aged MIKl-KO and WT animals in the time spent in the quadrant platform (Q4) are shown in the swimming heatmap tracks shown in Fig 4h. Aged Mlkl-KO mice travelled a larger distance and spent more time exploring in the target quadrant Q4 compared to aged WT animals (Fig $\mathbf{4 i}, \mathbf{j}$ ), without significant changes in the mean 
swimming speed between both groups (data not shown), demonstrating that loss of Mlkl prevents learning and memory loss associated with aging.

These results demonstrate that an age-associated increase in brain necroptosis induces degeneration of axons in the dentate gyrus of the hippocampus, thereby depressing synaptic transmission, and impairing hippocampal-dependent functions, such as learning and memory in aged mice.

\subsection{Pharmacologic inhibition of necroptosis reverts key signs of brain aging, improving hippocampal function and memory.}

To further explore the role of necroptosis in brain aging, we developed a pharmacologic strategy by using the selective RIPK3 inhibitor, GSK'872 (Salvadores \& Court 2020; Yang et al. 2017). In order to evaluate whether necroptosis inhibition reverts age-associated alterations, we designed a strategy in which GSK' 872 was systemically administrated in 23-month-old mice for only one month. Diffusion pumps were filled with vehicle or GSK'872, and intraperitoneally implanted to continuously diffuse the inhibitor (2 mg/kg GSK'872 at $0.11 \mu \mathrm{l} / \mathrm{hr}$ for 28 days). In order to evaluate whether GSK'872 systemically administrated is capable to cross the blood brain barrier, and then distributed into the brain, we performed pharmacokinetic studies. Measurable levels of GSK' 872 were detected in the brain $(187.6 \pm 17.11 \mathrm{~nm})$ and in plasma $(18.32 \pm 0.85 \mu \mathrm{m})$ samples collected from aged animals after $1 \mathrm{~h}$ of a single i.p. administration of $10 \mathrm{mg} / \mathrm{kg}$ of GSK'872. The efficiency of GSK' 872 treatment in the brain was evaluated by measuring phosphorylated RIPK3 (pRIPK3) levels in the hippocampus, demonstrating decreased pRIPK3 signal in the hilus of aged GSK'872-treated mice (Fig S6). Remarkably, aged mice treated with GSK' 872 showed decreased non-pNF staining in comparison to vehicle treated mice of the same age (Fig 5a). In fact, non-pNF levels in GSK'872 treated aged mice were comparable with those observed in adult animals (Fig $\mathbf{5 b}$ and Fig $\mathbf{3 c}$ ), demonstrating that one month of systemic inhibition of RIPK3 reverts signs of axonal degeneration associated with brain aging. Moreover, decreased microglia activation was also observed, indicating that a short-term treatment with GSK'872 is capable of reverting one of the main signs of brain inflammation associated with aging (Fig 5c, d).

Since RIPK3 is involved in several pathways that regulate cytokines secretion and the inflammatory response (Daniels et al. 2017; Kataoka et al. 2015; Orozco \& Oberst 2017) we performed Luminex High Performance Assay to detect changes in a pool of selected cytokines (Tables S1). Interestingly, the hippocampus of GSK'872 treated mice showed a pattern of cytokine levels highly similar to those observed in untreated adult mice (Fig 5e). Remarkably, this analysis reveals that RIPK3 inhibition mainly reduces pro-inflammatory cytokines, such as TNF- $\alpha$, IL-6, IL-12, and IFN- $\gamma$, reaching youthful-like cytokine levels equivalent to adult mice (Fig $5 \mathbf{f}$ ). A similar profile of decrease in pro-inflammatory cytokines was observed systemically in serum samples of GSK'872 treated mice, as was also noticed in the genetic model (Fig S5). Altogether, these results demonstrate 
that a short-term administration of GSK'872 can revert key hallmarks of brain aging, such as axonal damage and neuroinflammation, and might have a positive impact in hippocampal-dependent functions.

Extracellular electrophysiological recordings in the hippocampus of aged mice pharmacologically treated with the inhibitor of necroptosis indicated that aged GSK'872 treated mice present fEPSP equivalent to untreated adult mice (Fig 6a). Average traces showed significant differences between aged vehicle-treated mice versus those that received the RIPK3 inhibitor (Fig 6b,c). To evaluate the axonal contribution on this protective effect of GSK'872 on synaptic transmission, we calculated the facilitation index for each group. Facilitation index was significantly lower in the hippocampus of aged mice treated with GSK'872 compared with the aged-vehicle mice (Fig 6d,e), showing that late necroptosis inhibition can revert the loss of synaptic strength in aged mice, by promoting axonal function.

To further explore the impact of GSK'872 administration on hippocampal-dependent behavioral skills we evaluated spatial learning and memory in aged GSK'872-treated mice. Remarkably, RIPK3 inhibition significantly improved learning in aged mice from the second day of training (Fig 6f). Memory assessment indicated that aged mice with GSK' 872 treatment spent more time in the target quadrant and travelled larger distance in Q4 compared with aged vehicle-treated mice. Thus, RIPK3 inhibition is capable to recover aged mice from learning and memory impairment, suggesting the inhibition of necroptosis as an attractive target to improve memory in the elderly.

\subsection{Proteomic analysis in the hippocampus of aged mice with genetic and pharmacological inhibition of necroptosis reveals improvement in key hallmarks of aging and features of brain rejuvenation.}

In order to elucidate the specific molecular alterations underpinning our necroptosis-inhibitory approaches toward reducing aging phenotypes, we employed a state-of-the-art single-shot, label free quantitative proteomic approach. Our aim was to generate a correlative necroptotic proteomic profile in order to gain insight toward how necroptosis or, conversely, its inhibition influences neuronal integrity in aging-associated degeneration. To this end, hippocampus of adult and aged WT mice, aged MIkl-KO mice, and aged GSK'872-treated mice were subjected to single-shot label-free mass spectrometry (see workflow in Fig S8a), obtaining a high degree of coverage of the proteome with almost 7,000 proteins detected (Fig S8b).

To determine which molecular processes were likely correlative with the aging-induced necroptotic events, relative expression ratios were calculated and used to perform expression profile clustering analyses. We identified and isolated subsets of proteins exhibiting opposing directionality in expression between the "normal" aging proteome (aged vs adult animals) and the proteomes of animals subjected to genetic (aged MIkl-KO vs aged WT) or pharmacological (aged GSK'872 vs aged 
vehicle) inhibition of necroptosis (Fig S8 and Fig S9). In doing so, it was possible to reduce the number of possible correlative candidate proteins to 2,516 proteins whose expression increases and 2,307 which decrease in the aging hippocampus and where genetic and pharmacological modulation leads to a degree of reversion (Fig 7a). Contextualization of these necroptosis-correlative clusters through pathway analysis confirmed opposing directionality in the activation status of numerous biological processes between "normal" and necroptosis-targeted aged animals. These included several canonical pathways and biological function annotations previously implicated in normal aging (López-Otín et al. 2013; Kennedy et al. 2014) (see hallmarks of aging, Fig S10a) and in brain rejuvenation (Bouchard \& Villeda 2015; Wyss-Coray 2016) (Fig 7b and Fig S10b). Most of the molecular cascades belonging to these groups of pathways are typically associated with neurodegeneration and/or neuronal aging, including all the molecular cascades involved in synaptic mechanisms, senescence and cellular homeostasis, which are indeed affected in the normal aging proteome and recovered in the genetic and pharmacological models of necroptosis inhibition (Fig 7b).

In order to explore the contribution of necroptosis in age-associated neuronal dysfunction, we evaluated several biological functions affected by normal aging in our proteomic analysis. These biofunctions were classified considering their contribution in central brain functions, designated as neurodegeneration, synaptic function, cognitive and behavior, and neuronal development. It is interesting to note that the patterns of change of these biofunctions show opposing directionality in both the genetic and pharmacological approaches of necroptosis inhibition compared with normal aging in most of the biological functions analyzed (Fig 7c). Remarkably, key molecular and cellular functions associated with neurodegeneration, neuronal integrity and function, including cell survival and viability, cellular homeostasis, synapse development, cognition, motor dysfunction and cytoskeleton organization, showed a clear reversion in the context of pathway analysis in necroptosistargeted aging in comparison with normal aging (Fig 7c). This analysis demonstrated that the inhibition of necroptosis supports proper brain function in aged animals, improving key hallmarks of aging and restoring (in the case of our pharmacologic treatment with GSK'872) relevant functions involved in brain rejuvenation.

\subsection{Necroptosis inhibition induces synaptic-long term potentiation in aged mice.}

Among the cascades elucidated in our proteomic analysis with clear opposed profiles between normal aging and necroptosis inhibitory conditions, those classified as synaptic mechanisms showed the highest predicted z-score. Synaptic long-term potentiation (LTP) is the most common studied form of synaptic plasticity (Citri \& Malenka 2008), a key process directly related with learning and memory, and early impaired during aging (Lynch et al. 2006). The contribution of the synaptic LTP signaling at the level of individual molecules is visually illustrated in Fig 8a. These molecular changes are mostly prevented in the aging process of the MIkl-KO mice (red molecules) and partially reverted in the 
pharmacologic inhibition of necroptosis with GSK'872 in aged animals (Fig 8b). We therefore analyzed synaptic plasticity by studying LTP magnitude in hippocampal CA1-CA3 transmission. By the usage of a high-frequency stimulation protocol, we found that LTP induction was compromised in aged WT mice when compared to adult WT mice (Fig 8c). Surprisingly, adult MIkl-KO mice presented a higher LTP magnitude than the control WT group and the loss of Mlkl in aged mice restored LTP induction and maintenance beyond adult WT mice potentiation. Synaptic plasticity was also assessed in the pharmacological model of necroptosis inhibition in aged mice treated with GSK'872. Similar to KO experiments, we detected a reduction in LTP magnitude when we compared adult WT mice with aged vehicle-treated mice. Surprisingly, only one month of GSK'872 treatment was capable to improve LTP magnitude, reaching adult-like levels (Fig 8d), demonstrating that inhibition of necroptosis either by genetic knock-out of MIkl or by pharmacologic RIPK3 inhibition improved or restore synaptic plasticity in aged mice, a synaptic process that is crucial to support brain rejuvenation (Wyss-Coray 2016).

\subsection{Necroptosis activation contributes to the acquisition of the age-associated senescent phenotype in the hippocampus during aging.}

The accumulation of senescent cells in aged tissue, including the brain, is one of the most common feature of aging (Wang et al. 2009; Jurk et al. 2012; Moreno-Blas et al. 2019; Wiley \& Campisi 2021). As neuronal senescence has also been detected in the hippocampus of aged mice (Gorostieta-Salas et al. 2021), we aimed to determine whether necroptosis contributes to the establishment of the senescent phenotype of hippocampal neurons along aging. From our proteomic data, the molecular changes associated to senescence are mostly prevented in the aging process of the Mlkl-KO mice (green molecules) and again, as observed with other signaling pathways, partially reverted in the pharmacologic inhibition of necroptosis with GSK'872 in aged animals (Fig 9). Interestingly, the expression profile of two key proteins involved in the acquisition of the senescent phenotype and in the regulation of the senescence-associated secretory phenotype (SASP), which are CDKN1B and NFKB1 respectively (Pruitt et al. 2013; Bernal et al. 2014), is mainly reverted in both Mlkl-KO and GK'872-treated mice during aging (blue dotted insets, Fig 9a and Fig 9b). We therefore evaluated

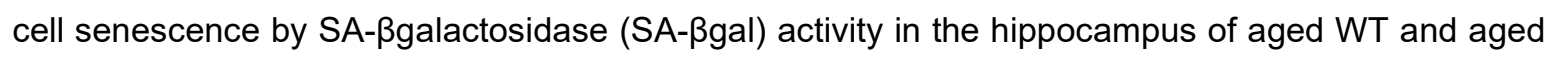
MIkl-KO mice. As was previously described, the accumulation of SA- $\beta$ gal positive neurons is mainly observed in the CA2/3 subfield of the hippocampus of aged mice (Gorostieta-Salas et al. 2021) (Fig 9c). SA-Bgal reactivity was significantly lower in aged Mlkl-KO mice in the CA2/3 area (Fig 9c, d). Cellular senescence was also evaluated in the pharmacological model of necroptosis inhibition in aged mice treated with GSK'872. A reduced SA- $\beta$ gal activity was also observed in the hippocampus of aged mice treated with the RIPK3 inhibitor compared with the vehicle treated animals (Fig 9e, f), reinforcing the data obtained from the proteomic analysis, which overall indicates that age-related activation of necroptosis contributes to the development of key pathological changes that are involved in brain aging. 
Together our findings support the conclusion that necroptosis activation participates in the development of the major causes of brain disfunction during aging, including axonal degeneration, neuroinflammation, synaptic dysfunction and cognitive disabilities. By contrast, the inhibition of necroptosis prevents these major features of brain aging, restoring synaptic transmission and axonal function in the hippocampal circuitry, thus improving learning and memory defects associated with aging. Furthermore, a short systemic inhibition of necroptosis was capable to revert age-associated hippocampal changes and restore learning and memory capacity to youthful levels.

\section{DISCUSSION}

Our findings reveal the involvement of necroptosis in the progression of $A x D$ in the hippocampus during normal aging, uncovering relevant implications of necroptosis activation for age-associated cognitive impairment, which might undermine healthy aging. Of note, most detrimental consequences of brain aging, including neuroinflammation, synaptic failure and hippocampal-dependent behavioral impairment were prevented through inhibition of necroptosis by genetic or pharmacological means. In our mouse model of aging, neurodegeneration appears to be restricted to axonal-enriched brain regions, including the corpus striatum, the cerebellum, and white matter regions of the spinal cord (Fig 1 and S1). Corresponding with this observation, neurodegeneration was also noted in hippocampal subfields recognized as the richest axonal projection regions, including DG-CA3-CA1 axonal pathways (Ropireddy et al. 2011; Wittner et al. 2007). Importantly, necroptosis activation was also confined to these axonal-enriched areas of the hippocampus, particularly in the DG (Fig 1), which is the only subfield that has been shown to present neuronal loss as a consequence of aging in humans (West 1993). The DG is a crucial area in the hippocampus supporting neuronal connectivity. It receives entorhinal cortex information which is transmitted to the CA3-CA1 neurons to complete the hippocampal circuitry. DG neurons, particularly mossy cells of the hilus, are extremely vulnerable to degenerative insults and injury (Santhakumar et al. 2000; Scharfman et al. 2001; Buckmaster \& Jongen-Rêlo 1999), whereas DG granule cells are more resistant (Sloviter 1987; Scharfman 2016). This evidence is aligned with our results showing increased necroptosis in hilar cells, and an early activation in the hilar fibers of the DG along with aging (Fig 2). Moreover, it has been shown that this confined subdivision of the hippocampus where mossy cells reside is also highly vulnerable to the effects of aging (West 1993), compromising DG connectivity with other subfields of the hippocampus (Smith et al. 2000; Amani et al. 2021), and increased susceptibility to failure in memory encoding and retrieval in aged individuals (Hainmueller \& Bartos 2020; Dillon et al. 2017). These observations suggest that the restricted activation of necroptosis in DG hilar cells and axons may contribute to the vulnerability of this area to degenerate along with aging, to adversely impact synaptic function and hippocampal-dependent behavioral performance (Yassa et al. 2011). 
The functional role of necroptosis as a crucial pathogenic determinant in experimental models of human diseases has been of increasing interest in the field, focusing on the central effectors of necroptosis, RIPK3 and MLKL, as possible markers of disease progression and severity in humans (Choi et al. 2019; Picon et al. 2021). Increased pMLKL levels in aged mice suggests that necroptosis could be considered as a biomarker of aging progression. To further address the involvement of necroptosis in the harmful effects of aging in the hippocampus, we evaluated AxD in the DG, neuroinflammation, synaptic function and memory capabilities in aged Mlkl-deficient mice. Of note, aged Mlkl-KO mice presented a youthful phenotype in the hippocampus, reaching levels of $A x D$ comparable with those observed in the old mice group (Fig 3a, b). According to the survival curve comparison between mice and humans (Hagan 2017), old mice (12-15 months old) are equivalent to humans between 38 to 47 years old, a life stage in which basal levels of pMLKL were only slightly detected in the hippocampus (Fig 2). Interestingly, there is no incidence of dementia and cognitive impairment at this stage of life in humans going through a normal aging process (without inherited conditions and/or associated pathologies) (Rocca et al. 2011), suggesting that maintaining necroptosis at lower activation levels could prevent age-associated pathological conditions.

As a chronic inflammatory condition, aging also influences the inflammatory status of the brain (Kennedy et al. 2014; Luo et al. 2019), mainly through microglia activation and increase of proinflammatory cytokines (Barrientos et al. 2015; Schuitemaker et al. 2012; von Bernhardi et al. 2015). Among the pro-inflammatory cytokines analyzed in the hippocampus of aged MIkl-KO mice only IL12 decreased, reaching levels comparable with adult mice (Fig 3f). IL-12 is produced in the brain by microglia, and required for IFN-y and TNF- $\alpha$ production, two master inflammatory cytokines (Trinchieri 2003; Aloisi et al. 1997). The increased expression of IL-12 in the brain has been associated with spontaneous neurological disorders in aged mice (Hofer et al. 2004). By contrast, the IL12-KO mouse exhibits lower levels of microglia activation and reduced neurodegeneration in an excitotoxicitymediated injury model (Chen et al. 2004). Moreover, increased levels of the anti-inflammatory cytokines, IL-2 and IL-10, were specifically detected in aged MIkl-KO mice (Fig 3f), suggesting that MLKL could also act as a repressor of anti-inflammatory cytokine expression under necroptosis activated conditions. Interestingly, IL-10 inhibits the production of IL-12 (Lobo-Silva et al. 2016), which accompanied with the IL-12 decrease observed in the aged MIkl-KO mice generates a positive antiinflammatory feedback loop in conditions of MIkl deficiency, limiting neuroinflammation.

It is well documented that neurodegeneration contributes to cognitive decline during normal aging (Bettio et al. 2017; Rosczyk et al. 2008; Tegeler et al. 2016). Hippocampal-dependent function are determined by accurate electrophysiological signals. Loss of Mlkl in aged mice produces changes in the hippocampal circuit that prevent the loss of synaptic strength observed in normal aging (Fig $\mathbf{4 d}$ ). The increased facilitation index observed in aged WT mice (Fig 4f) is an indicator of a decreased neurotransmitter release probability from the presynaptic compartment, which confirms that aged mice present hippocampal axonal failure. Remarkably, loss of MIkl showed a significant decrease in 
paired-pulse facilitation in aged mice, indicating that necroptosis is in fact contributing to the axonal defects shown in aged mice. Electrophysiological results were supported by improved learning and memory performance in aged Mlkl-KO mice, demonstrating that altogether axonal protection, controlled anti-inflammatory status, as well as synaptic transmission restoration in Mlkl deficiency favor youthful-like behavior in aged mice. These results support the notion that lower levels of necroptosis activation improves key cognitive functions that might positively impact the quality of life, favoring healthy aging.

Multiple efforts have been made in the field of geroscience to understand the aging process and to consider it as a therapeutical target to improve healthspan (Kennedy et al. 2014; Hansen \& Kennedy 2016; Hodgson et al. 2020). The brain and peripheral organs share common biological mechanisms of aging (López-Otín et al. 2013). Thus, the development of anti-aging drugs that improve cognitive function and hence the quality of life in old age could have a significant potential at improving healthspan. Our pharmacological strategy to systemically inhibit RIPK3 with GSK'872 in aged mice demonstrated to be extraordinarily effective on reverting $A x D$, neuroinflammation and hippocampusdependent functional impairment (Fig 5 and Fig 6). Interestingly, the evaluation of a pool of cytokines showed reduced levels of most of the pro-inflammatory cytokines analyzed in the hippocampus of aged mice treated with GSK'872, including TNF- $\alpha$, IL-6, IL-12, and IFN-Y (Fig 5e-f), indicating that systemic inhibition of necroptosis impacts the inflammatory status of the brain, thereby diminishing neuroinflammation. Moreover, the analysis of serum cytokine levels showed the same regulatory profile as the brain, demonstrating that systemically, the inflammatory condition of aged mice is maintained in a youthful-like state under necroptotic inhibitory terms.

Our quantitative proteomic analysis allowed us to generate a correlative necroptotic proteomic profile and to evaluate whether necroptosis inhibition influences key networks involved in brain aging. The analysis demonstrated that about 7,000 proteins changed their expression profile as a consequence of aging (Fig 7 and Fig S9), of which 2,516 shown to be upregulated and 2,307 were downregulated under necroptosis inhibitory conditions in the hippocampus of aged mice (Fig S9). From these clustered profiles we were capable to contextualize these proteomic changes by associated them with related canonical pathways and biological function annotations. The pathways analysis unveiled key biological processes with opposed directionality between "normal" and necroptosis-targeted aging. This unbiased analysis demonstrated that key hallmarks of aging, including synaptic function, mitochondrial dysfunction, stress response, cellular senescence, deficient nutrient sensing, altered metabolism, and others (Kennedy et al. 2014; López-Otín et al. 2013), are positively regulated under necroptosis inhibitory conditions (Fig S8), which may have potential impact in life quality by promoting healthy aging. Interestingly, our pharmacological approach demonstrated a similar trend in effect over the proteome as the genetic model, suggesting that necroptosis inhibition could be an attractive therapeutic strategy to develop geroprotector agents to slow aging. In fact, results obtained from our proteomic analysis indicated that GSK'872 treatment influences most of the different hallmarks of 
aging, an inclusion criteria that is currently recognized to accept novel molecule candidates as geroprotectors (Partridge et al. 2020). Furthermore, inhibition of necroptosis demonstrated ability to modulate several brain functions implicated in brain rejuvenation (Wyss-Coray 2016) (Fig 7 and Fig S10), including those elucidated from the proteomic analysis and experimentally evaluated, such as synaptic plasticity and neuronal senescence (Fig 8 and Fig 9), which added to the behavioral results on learning and memory, reinforced the potential of GSK' 872 as a candidate drug to protect against age-associated brain dysfunction, including memory decline. Moreover, other interesting pathways were also demonstrated to be positively modulated by necroptosis inhibition (see Fig S11-S14 for individual molecular changes and proteomic profiles). These pathways include synaptogenesis signaling, calcium signaling, CREB signaling in neurons, and others; most of them highly implicated in the maintenance of neuronal homeostasis and functioning, and consequently in brain-dependent functions, including cognitive and behavioral processes (Zia et al. 2021; Thibault \& Landfield 1996; Nikoletopoulou \& Tavernarakis 2012; Yu et al. 2017).

Overall, our study demonstrates that necroptosis contributes to the age-associated deterioration of axonal integrity and function, affecting hippocampal neuronal connectivity, and consequently the cognitive function of aged mice. Our results from the pharmacological intervention propose necroptosis inhibition as an interesting and novel therapeutic target to counteract the deleterious effects of aging, thus increasing healthspan and potentially delaying the onset of a range of agerelated disabilities.

\section{EXPERIMENTAL PROCEDURES}

\subsection{Animals}

Wild-type (WT) C57BL/6J mice of different ages were purchase from the Jackson Laboratory and maintained in the Universidad Mayor animal facility. Aging groups were established as follow: adult (3-6 month), old (12-15 month) and aged mice (more than 20 month). Mlkl knockout mice (MIkl-KO) were kindly provided by Dr Douglas Green (St. Jude Children's Research Hospital, Memphis, TN, USA) and have been previously produced and described (Murphy et al. 2013). The age range was selected in equivalence with the human life phases (Flurkey et al. 2007; Hagan 2017). Animals were kept under standard conditions of light and temperature and were feed with food and water ad libitum in the Animal Facility of the Sciences Faculty of the Mayor University. The research protocol no. 222017 was approved by the Animal Care and Use Scientific Ethic Committee of the Mayor University.

\subsection{Osmotic pump implantation}

Micro-osmotic pumps (Alzet, model 1004) containing the RIPK3 inhibitor GSK'872 (Tocris) (2 mg/kg) were surgically implanted in the peritoneal cavity of 23-moth-old. The pump allows a constant flux of 
the drug at $0.11 \mu \mathrm{l} / \mathrm{hr}$ for 28 days. One-month post-surgery, mice (24-month-old) were subjected to behavioral test to evaluate memory and then tissue was extracted for further analyses.

\subsection{Quantification of GSK'872 in brain and plasma}

Brain and plasma samples were obtained from aged mice treated for $1 \mathrm{~h}$ with GSK' 872 (10mg/kg i.p.). The bioanalysis of plasma and brain samples was conducted by LC-MS/MS with a QTRAP 4500 triple quadrupole mass spectrometer (Applied Biosystems SCIEX) in the negative ion mode and interfaced with an Ekspert ultraLC 100-XL UHPLC System (Eksigent). Calibration standards (0.003 to $10 \mu \mathrm{M})$ and quality controls $(0.02,0.2$ and $2.0 \mu \mathrm{M})$ were prepared from naïve mouse plasma in parallel with mouse plasma study samples $(60 \mu \mathrm{L})$ by precipitation with three volumes of ice-cold acetonitrile containing $20 \mu \mathrm{M}$ of theophylline. The precipitated samples were centrifuged at $6,100 \mathrm{~g}$ for $30 \mathrm{~min}$ at $4^{\circ} \mathrm{C}$. Following centrifugation, an aliquot of each supernatant was transferred to an autosampler vial and diluted with two volumes of aqueous mobile phase $(0.2 \%$ formic acid in water). Samples were injected onto a reverse phase analytical column (YMC Triart C18; 2.0 x 50 mm; $1.9 \mu \mathrm{m}$; YMC CO) and eluted with a gradient of $0.2 \%$ formic acid in Acetonitrile. GSK' 872 was monitored by a multiple reaction monitoring (MRM) experiment using an Analyst software (v1.6.2, Applied Biosystems SCIEX). Quantitation was conducted using a MultiQuant software (v2.1, Applied Biosystems SCIEX) and the resulting calibration curve was fitted with a linear regression and $1 / x$ weighting. The lower limit of quantitation (LLOQ) was $0.010 \mu \mathrm{M}$.

\subsection{Immunohistochemistry}

Mice were deeply anesthetized with isoflurane and intracardially perfused with isotonic saline followed by $4 \%$ paraformaldehyde. Brains were dissected, postfixed overnight in $4 \%$ paraformaldehyde at $4{ }^{\circ} \mathrm{C}$, and then incubated in $30 \%$ sucrose. Tissue was cryoprotected in optimal cutting temperature compound (OCT, Tissue-Tek) at $-20^{\circ} \mathrm{C}$ and serial sagittal sections of $20 \mu \mathrm{m}$ thickness were obtained using a cryostat (Leica, CM1860). Brain sections were pre-mounted on positively charged slides and washed in TBS. After antigen retrieval $\left(80{ }^{\circ} \mathrm{C}, 30 \mathrm{~min}, 10 \mathrm{mM}\right.$ citrate buffer, $\left.\mathrm{pH} 6.0\right)$, sections were blocked in TBSB (TBS, $5 \%$ BSA and $0.25 \%$ Triton X-100), and then incubated overnight at $4{ }^{\circ} \mathrm{C}$ with the following primary antibodies: pMLKL (1:200, phospho S345, Abcam, ab196436), neurofilament pan-axonal cocktail (1:250, SMI312 clone, Biolegend), non-phosphorylated neurofilament (1:250, SMI32 clone, Biolegend), and Iba1 (1:500, Wako Chemicals, 016-26721). Sections were washed to remove the excess of primary antibody and incubated with the appropriate Alexa-Fluor secondary antibodies for $2 \mathrm{~h}$ at $25^{\circ} \mathrm{C}$ (1:1000, Thermo Fisher Scientific). The excess of secondary antibody was washed, and the sections were coverslipped with Fluoromount-G antifade mounting medium (EMS, 17984-25) containing DAPI staining for nuclei detection (Thermo Fisher Scientific). For Fluoro-Jade C staining, brain tissue was mounted on positively charged slides and rehydrated on decreasing concentrations of ethanol. After rehydration, the tissue was pre- treated for 10 min in potassium 
permanganate and then incubated for $10 \mathrm{~min}$ in the dark at $25{ }^{\circ} \mathrm{C}$ in Fluoro-Jade $\mathrm{C}$ and DAPI (Biosensis, TR-100-FJ). The tissue was then washed with water and let dry overnight. The next day the slides were cleared in xylene and coverslipped with DPX-new mounting solution (Merck Millipore). The anti-pMLKL antibody purchase from Abcam (ab196436) was KO-validated by comparison of the immunodetected signal in aged WT mice with the MIkl-KO mice in the hippocampus (Fig S7). Immunostained sections were scanned in a Leica DMi8 Fluorescence Microscope fully automatized.

\subsection{Histological Analysis}

Free-floating sections were processed for immunohistochemistry as previously described (Oñate et al. 2020). Briefly, slices were quenched with $0.3 \% \mathrm{H}_{2} \mathrm{O}_{2}$ for $30 \mathrm{~min}$, blocked with $5 \% \mathrm{BSA}$ and $0.2 \%$ triton $\mathrm{X}-100$ for $2 \mathrm{~h}$ and incubated with primary antibody (rabbit anti-pMLKL, 1:200 Abcam) overnight at $4{ }^{\circ} \mathrm{C}$. Then, sections were washed with $0.1 \mathrm{M}$ PBS and incubated with secondary biotinylated antibody (goat anti-rabbit, 1:500 Vector Laboratories) for $2 \mathrm{~h}$ at RT. After washing, slices were incubated with avidin-biotin-peroxidase complex (Vector Laboratories) for $1 \mathrm{~h}$ at RT followed by 0.1 M PBS washes and developed with 3,3-diaminobenzidine (DAB, Sigma-Aldrich). Finally, sections were co-stained with Nissl (cresyl-violet staining) to detect nuclei and with Eriochrome-C staining for myelinated-axons detection, and finally mounted on glass slides with Entellan medium (Merck).

\subsection{Image Analysis}

Fluorescent images were analyzed using the Image $\mathrm{J}$ software from the $\mathrm{NIH}$, USA. Colocalization image analysis was performed with the JACoP plugin (Bolte \& Cordelières 2006). Mander's coefficient M2 was used to estimate the percentage of PMLKL signal over Map2 or Iba1 labeling in human samples. Axonal degeneration index (DI) was measured in pan-axonal immunostained images as the ratio of the area of fragmented axons against the total axonal area (intact + fragmented particles) in the hilus using the particle analyzer algorithm of Image J. Fragmented and intact axonal particles were estimated by defining area and circularity of the particles (fragmented: $<20 \mu \mathrm{m}^{2}$ and $0.3 \leq 1$ circularity; intact: $\geq 20 \mu \mathrm{m}^{2}$ and $0<0.3$ circularity) (Arrázola et al. 2019).

\subsection{Luminex Assay}

Cytokines levels were analyzed by Luminex Mouse Discovery Assay (R\&D Systems, MN, USA) using a self-designed panel of 12 selected cytokines (plate code: LXSAMSM-12), based on color-coded beads, pre-coated with analyte-specific capture antibodies that permits simultaneous analysis of the analytes. Table S1 detailed cytokines and chemokines of the panel, bead region, sensitivity, and the main functions. Analysis and detection were performed in a Dual-laser flow-based detection instrument, Luminex 200 analyzer by Proyecto Luminex, Programa de Virología, Redeca, ICBM, Facultad de Medicina, Universidad de Chile.

\subsection{Electrophysiology}


Electrophysiological recordings were performed as described before (Carvajal et al. 2018). Briefly, transverse slices $(400 \mu \mathrm{m})$ from the dorsal hippocampus were cut under cold artificial cerebrospinal fluid (ACSF, in mM: $124 \mathrm{NaCl}, 2.6 \mathrm{NaHCO}_{3}, 10 \mathrm{D}$-glucose, $2.69 \mathrm{KCl}, 1.25 \mathrm{KH}_{2} \mathrm{PO}_{4}, 2.5 \mathrm{CaCl}_{2}, 1.3$ $\mathrm{MgSO}_{4}$, and 2.60 NaHPO 4 ) using a Vibratome (BSK microslicer DTK-1500E, Ted Pella, Redding, CA, USA) and incubated in ACSF for 1 hour at room temperature. In all experiments, $10 \mu \mathrm{M}$ PTX was added to suppress inhibitory GABAA transmission. Slices were transferred to an experimental chamber ( $2 \mathrm{ml}$ ), superfused ( $3 \mathrm{ml} / \mathrm{min}$, at room temperature) with gassed ACSF (using $95 \% \mathrm{O}_{2} / 5 \%$ $\mathrm{CO}_{2}$ ) and visualized by trans-illumination with a binocular microscope (Amscope, Irvine, CA, USA). To evoke field excitatory post synaptic potentials (fEPSPs), Schaffer collaterals were stimulated with bipolar concentric electrodes (Tungsten, $125 \mu \mathrm{m}$ OD diameter, Microprobes) connected to an isolation unit (Isoflex, AMPI, Jerusalem, Israel). The stimulation was performed in the stratum radiatum within 100-200 $\mu \mathrm{m}$ from the recording site. Recordings were filtered at $2.0-3.0 \mathrm{kHz}$, sampled at $4.0 \mathrm{kHz}$ using an A/D converter (National Instrument, Austin, TX, USA), and stored with the WinLTP program. The basal excitatory synaptic transmission was measured using an input/output curve protocol with $10 \mathrm{~s}$ of interval between stimuli. Data were collected and analyzed offline with pClamp 10 software (Molecular Devices, San Jose, CA, USA). To generate LTP, we used highfrequency stimulation (HFS) protocol, which consisted of 3 trains at $100 \mathrm{~Hz}$ of stimuli with an intertrain interval of $10 \mathrm{~s}$. Data were collected and analyzed offline with pClamp 10 software (Molecular Devices, San Jose, CA, USA).

\subsection{Learning and memory test}

Morris water maze (MWM) navigation task was performed to evaluated spatial memory and learning in adult vs aged WT, MIkl-KO and GSK'872-treated mice. Animals were trained in a 1.2-m-diameter circular pool (opaque water, $50 \mathrm{~cm}$ deep) filled with $19-21^{\circ} \mathrm{C}$ water. A submerged 11.5 -cm platform $(1 \mathrm{~cm}$ below the surface of water, invisible to the animal) was used for training, with a maximum trial duration of $60 \mathrm{~s}$; the mice remained on the platform for $10 \mathrm{~s}$ at the end of each trial. Each animal was trained to locate the platform for 4 consecutive days following external cues (learning curve), 4 times per day. The test was performed on the fifth day by removing the platform, and swimming was monitored to measure the latency time required to reach the platform and the time spent in each quadrant and in the target quadrant (Q4), and the travelled distance within Q4. Both the learning curve and the test were tracked using an automatic tracking system (ANY-maze video tracking software, Stoelting Co, Wood Dale, IL, USA) to obtain the parameters measured. After testing, the mouse was gently removed from the pool and returned to its cage. Mean heatmaps were generated by the Anymaze software to represent the time spent by differents cohorts of mice in a particular location of pool.

\subsection{Senescence-associated beta-galactosidase (SA-ßgal) activity}


Histochemical detection of SA- $\beta$ gal activity were performed as was described before (DebacqChainiaux et al. 2009). Briefly, SA-ßgal activity was determined by incubation with $1 \mathrm{mg} / \mathrm{mL}$ of solution of 5-bromo-4-chloro-3-indolyl $\beta$-d-galactopyranoside in $0.04 \mathrm{M}$ citric acid/sodium, $0.005 \mathrm{M} \mathrm{K}_{3} \mathrm{FeCN}_{6}$, $0.005 \mathrm{M} \mathrm{K}_{4} \mathrm{FeCN}_{6}, 0.15 \mathrm{M} \mathrm{NaCl}$, and $0.002 \mathrm{M} \mathrm{MgCl}_{2}$ diluted in phosphate-buffered saline $(\mathrm{pH} 6)$ for 16h. After the incubation, hippocampal slices were washed with TBS and mounted in superfrost microscope slides (TermoFisher, 6776214) using Fluoromount-G (TermoFisher, 00-4958-02). Images were taken with Nikon Eclipse E200 optic microscope with 4X and 10X objective magnification. ImageJ software was used to process the images. Positive area for SA- $\beta$ gal activity was measured and representative images are shown.

\subsection{Statistical Analysis}

Statistical significance was stablished at $p<0.01$ by one-way ANOVA with Tukey's post-test or twoway ANOVA for multiple comparisons. The analysis was performed using GraphPad Prism Software v8.0 and performed with data obtained from $\geq 3$ independent experiments for image analyses and $n$ $\geq 8$ animals for behavioral test.

\subsection{Label-free proteomics}

\section{S-Trap processing of samples}

Samples were processed using S-trap mini protocol (Protifi) (for 310 ug and 110 ug samples) and Strap micro protocol (for low conc samples) as recommended by the manufacturer with little modification. After, application of the samples on the S-trap mini spin column, trapped proteins were washed 5 times with S-TRAP binding buffer. A double digestion with trypsin (1:40) was carried out first overnight at $37^{\circ} \mathrm{C}$ in TEAB at a final concentration of $50 \mathrm{mM}$, and then for another $4 \mathrm{hrs}(1: 40)$ in $50 \mathrm{mM}$ TEAB. Elution of peptides from S-trap mini spin column was achieved by centrifugation at $1000 \times \mathrm{g}$ for $1 \mathrm{~min}$ by adding $50 \mathrm{mM}$ TEAB, then $0.2 \%$ aqueous formic acid and finally $50 \%$ acetonitrile/ $0.2 \%$ formic acid. Resulting tryptic peptides were pooled, dried, and quantified using Pierce Quantitative fluorometric Peptide Assay (Thermo Scientific).

\section{LC-MS methods}

$1.5 \mu \mathrm{g}$ peptide was analysed per sample. Samples were injected onto a nanoscale C18 reversephase chromatography system (UltiMate 3000 RSLC nano, Thermo Scientific) then electrosprayed into an $Q$ Exactive Plus Mass Spectrometer (Thermo Scientific). For liquid chromatography buffers were as follows: buffer A $(0.1 \%$ formic acid in Milli-Q water $(\mathrm{v} / \mathrm{v}))$ and buffer $\mathrm{B}(80 \%$ acetonitrile and $0.1 \%$ formic acid in Milli- $Q$ water $(\mathrm{v} / \mathrm{v})$. Sample were loaded at $10 \mu \mathrm{L} / \mathrm{min}$ onto a trap column $(100 \mu \mathrm{m}$ $\times 2 \mathrm{~cm}$, PepMap nanoViper C18 column, $5 \mu \mathrm{m}, 100 \AA$, Thermo Scientific) equilibrated in $0.1 \%$ trifluoroacetic acid (TFA). The trap column was washed for $5 \mathrm{~min}$ at the same flow rate with $0.1 \%$ TFA then switched in-line with a $\mu$ PAC C18 nano-LC column $(200 \mathrm{~cm}$, inter-pillar distance- $2.5 \mu \mathrm{m}$, pore size- 100-200 A, PharmaFluidics). The peptides were eluted from the column at a constant flow 
rate of $300 \mathrm{nl} / \mathrm{min}$ with a linear gradient from $3.8 \%$ buffer $B$ to $12.5 \%$ buffer $B$ in 22 mins, then from $12.5 \%$ buffer $B$ to $41.3 \%$ buffer $B$ in 95 mins, then from $41.3 \%$ buffer $B$ to $61.5 \%$ in 23 mins and finally to $100 \%$ buffer $B$ in 10 mins. The column was then washed with $100 \%$ buffer $B$ for 10 min and reequilibrated in $1 \%$ buffer $B$ for 38 mins. Two blanks were run between each sample to reduce carryover. The column was kept at a constant temperature of $50^{\circ} \mathrm{C}$. The data was acquired using a uPACcompatible easy spray emitter source operated in positive mode with spray voltage at $2.2 \mathrm{kV}$, and the ion transfer tube temperature at $275^{\circ} \mathrm{C}$. The MS was operated in DIA mode. A scan cycle comprised a full MS scan (m/z range from 345-1155), with RF lens at $60 \%$, AGC target 3E6, orbitrap resolution 70,000 , maximum injection time at 200 ms and source fragmentation disabled. The MS survey scan was followed by MS/MS DIA scan events using the following parameters: collision energy mode set to linear with a normalized HCD collision energy set to 25 , orbitrap resolution 17500 , first fixed mass $200 \mathrm{~m} / \mathrm{z}$, AGC target 3E6, maximum injection time $55 \mathrm{~ms}$, isolation windows were variable from 5-66 $\mathrm{m} / \mathrm{z}$. The inclusion list (DIA windows) and windows widths are shown in Table S2. Data for both MS and MS/MS scans were acquired in profile mode. Mass accuracy was checked before the start of samples analysis.

\subsection{Analysis of proteomic data}

\section{Data filtering and generation of expression ratios}

Raw data files from single-shot label-free experiments were converted into Microsoft Excel workbooks and utilised to generate ratios of protein expression within each animal relative to mean expression of $n=4$ control animals within each comparison (ie. adult: adult (expression ratio=1), aged: adult, MlklKO: aged-WT; aged GSK'872: aged vehicle). Proteins identified by fewer than 2 unique peptides were excluded from subsequent analyses (Figure S8) in order to ensure maximum identification confidence. Relative expression ratios per study (ie. aged vs. adult, MIkl-KO vs. aged WT, and aged GSK'872 vs. aged vehicle) were used for subsequent expression profile clustering analyses. UniProt Accession numbers of proteins identified by 2 or more unique peptides with accompanying expression ratios generated as described above were subjected to expression profile clustering in BioLayout Express $^{3 D}$. BioLayout utilises a user-determined Pearson correlation and the Markov Clustering Algorithm to cluster input data based on user-determined parameter(s) (Enright 2002; Theocharidis et al. 2009). Pearson correlation was set to 0.97 to cluster datasets into distinct subsets based on similarity in expression profile. Discrete clusters exhibiting biologically relevant expression profilesie. opposing directionality between aged versus both MLKL-KO and GSK'872 expression ratios (Fig S8 and Fig S9) were identified and exported as .txt files containing and identifier column and expression ratios, for subsequent analyses in IPA.

\section{Ingenuity Pathway Analysis (IPA)}

The Ingenuity Pathway Analysis (IPA) application (Ingenuity Systems, Silicon Valley, CA) was used to visualise and explore the cellular and molecular pathways that may have been altered as result of 
genetic (MIKl-KO) or pharmacological (GSK'872) inhibition of necroptosis. Without user-directed manipulation, IPA's statistical predictions and annotations are approximately $90 \%$ based off on peerreviewed publications; the remaining $10 \%$ of stored interactions have been identified by other in silico techniques. The analyses were performed only using experimentally reported interactions published in peer-reviewed publications stored within the "hand-curated" and continually updated Ingenuity Knowledge database (Ingenuity Systems, Silicon Valley, CA). For more information on the computational methodology underpinning IPA, please refer to http://www.ingenuity.com/.

Prior to all analyses within IPA, input datasets comprising, as described above, mean expression ratios of $n=4$ animals per experimental group, were converted to fold-change values, and a $\pm 20 \%$ cutoff in expression change respective to control was applied within each respective study. Individual analyses of aged vs. adult, Mlkl-KO vs. WT, and GSK'872 vs. vehicle were performed prior to a comparative analysis in order to gain insight into potential biological networks distinguishing "normal" versus "necroptosis-inhibited" aging processes.

For canonical pathway analysis, p-values of canonical pathway scores and subsequent ranking for all analyses performed in this study were derived from a Fisher's Exact Test calculating overlap between molecules in each respective input dataset and number of molecules comprising canonical pathway as defined by the Ingenuity Systems Database. Predicted activation z-scores were calculated by weighing the predicted expression change of target molecules as defined by Ingenuity Knowledge Database against the actual expression change of target molecules reported in input dataset. An activation z-score $>2$ or $<-2$ is considered statistically significant (Ingenuity Systems, Silicon Valley, CA). Constituent molecules within pathway were colourized with intensity of colour corresponding to magnitude of change.

For diseases and functions analysis, predicted activation z-scores of associated downstream diseases and functions were calculated by weighing the predicted expression change of target molecules associated with specific "diseases or functions" annotation as defined by Ingenuity Knowledge Database against the actual expression change of target molecules reported in input datasets. An activation z-score $>2$ or $<-2$ is considered statistically significant. $P$-values of overlap is derived from a Fisher's exact test were derived from a Fisher's Exact Test calculating overlap between molecules in each respective input dataset and number of molecules comprising the known interactome of each regulator as defined by Ingenuity Systems Database. In graphical format, target molecules present within each proteomic dataset predicted to be activated or inhibited to mediate the associated "diseases or functions" annotation were visualised in relation to their associated predicted regulator and were colourised with intensity of colour corresponding to magnitude of change.

\section{Funding and acknowledgement}

This work was supported by grants from the Geroscience Center for Brain Health and Metabolism, FONDAP- 15150012 (to FAC and CC), Fondo Nacional de Desarrollo Científico y Tecnológico 
(FONDECYT) N 1150766 (to FAC), Michael J Fox Foundation for Parkinson's Research 17303 (to FAC), Agencia Nacional de Investigación y Desarrollo (ANID) FONDECYT Postdoctorado $N^{\circ}$ 3180313 (to MSA), BBSRC ISP funding (BBS/E/D/10002071) (to TMW and SLE), BBSRC EastBio (to SI), EMC (to RAK), Programa de Apoyo a Centros con Financiamiento Basal AFB 170004 (to SB), Fondo Nacional de Desarrollo Científico y Tecnológico (FONDECYT) N 1190620 (to WC), Center for Excellence in Science and Technology AFB 170005, PFB 12/2007 (to WC), Fondo Nacional de Desarrollo Científico y Tecnológico (FONDECYT) N 1200255 to CC, and a PhD fellowship by ANID (to $\mathrm{ML}$ ).

\section{References}

Aburto JM, Villavicencio F, Basellini U, Kjærgaard S \& Vaupel JW (2020) Dynamics of life expectancy and life span equality. Proc. Natl. Acad. Sci. U. S. A. 117, 5250-5259. Available at: https://zenodo.org/record/3571095 [Accessed April 20, 2021].

Agüero-Torres H, Thomas VS, Winblad B \& Fratiglioni L (2002) The impact of somatic and cognitive disorders on the functional status of the elderly. J. Clin. Epidemiol. 55, 1007-1012.

Aloisi F, Penna G, Cerase J, Menéndez Iglesias B \& Adorini L (1997) IL-12 production by central nervous system microglia is inhibited by astrocytes. J. Immunol. 159.

Amani M, Lauterborn JC, Le AA, Cox BM, Wang W, Quintanilla J, Cox CD, Gall CM \& Lynch G (2021) Rapid Aging in the Perforant Path Projections to the Rodent Dentate Gyrus. J. Neurosci. 41, JN-RM-2376-20.

Arrazola M \& Court F (2019) Compartmentalized necroptosis activation in excitotoxicity-induced axonal degeneration: a novel mechanism implicated in neurodegenerative disease pathology. Neural Regen. Res. 14, 1385. Available at: http://www.ncbi.nlm.nih.gov/pubmed/30964063 [Accessed April 23, 2019].

Arrázola MS, Saquel C, Catalán RJ, Barrientos SA, Hernandez DE, Martínez NW, Catenaccio A \& Court FA (2019) Axonal Degeneration Is Mediated by Necroptosis Activation. J. Neurosci. 39, 3832-3844. Available at: http://www.jneurosci.org/lookup/doi/10.1523/JNEUROSCI.088118.2019 [Accessed April 23, 2019].

Barnes CA \& McNaughton BL (1980) Physiological compensation for loss of afferent synapses in rat hippocampal granule cells during senescence. J. Physiol. 309, 473-485. Available at: http://doi.wiley.com/10.1113/jphysiol.1980.sp013521.

Barrientos RM, Kitt MM, Watkins LR \& Maier SF (2015) Neuroinflammation in the normal aging hippocampus. Neuroscience 309, 84-99. 
Bernal GM, Wahlstrom JS, Crawley CD, Cahill KE, Pytel P, Liang H, Kang S, Weichselbaum RR \& Yamini B (2014) Loss of Nfkb1 leads to early onset aging. Aging (Albany. NY). 6, 931-942. Available at: https://pubmed.ncbi.nIm.nih.gov/25553648/ [Accessed October 18, 2021].

von Bernhardi R, Eugenín-von Bernhardi L \& Eugenín J (2015) Microglial cell dysregulation in brain aging and neurodegeneration. Front. Aging Neurosci. 7, 1-21.

Bettio LEB, Rajendran L \& Gil-Mohapel J (2017) The effects of aging in the hippocampus and cognitive decline. Neurosci. Biobehav. Rev. 79, 66-86.

Bian P, Zheng X, Wei L, Ye C, Fan H \& Cai Y (2017) MLKL Mediated Necroptosis Accelerates JEVInduced Neuroinflammation in Mice. 8, 1-10.

Bock P, Spitzbarth I, Haist V, Stein VM, Tipold A, Puff C, Beineke A \& Baumgärtner W (2013) SpatioTemporal Development of Axonopathy in Canine Intervertebral Disc Disease as a Translational Large Animal Model for Nonexperimental Spinal Cord Injury. Brain Pathol. 23, 82-99. Available at: http://doi.wiley.com/10.1111/j.1750-3639.2012.00617.x [Accessed August 3, 2020].

Bolte S \& Cordelières FP (2006) A guided tour into subcellular colocalization analysis in light microscopy. J. Microsc. 224, 213-32. Available at: https://pubmed.ncbi.nlm.nih.gov/17210054/ [Accessed August 30, 2021].

Bouchard J \& Villeda SA (2015) Aging and brain rejuvenation as systemic events. J. Neurochem. 132, 5-19. Available at: https://onlinelibrary.wiley.com/doi/10.1111/jnc.12969.

Buckmaster PS \& Jongen-Rêlo AL (1999) Highly specific neuron loss preserves lateral inhibitory circuits in the dentate gyrus of kainate-induced epileptic rats. J. Neurosci. 19, 9519-9529. Available at: https://pubmed.ncbi.nlm.nih.gov/10531454/ [Accessed July 1, 2021].

Burke SN \& Barnes CA (2006) Neural plasticity in the ageing brain. Nat Rev Neurosci 7, 30-40. Available at: http://dx.doi.org/10.1038/nrn1809.

Caccamo A, Branca C, Piras IS, Ferreira E, Huentelman MJ, Liang WS, Readhead B, Dudley JT, Spangenberg EE, Green KN, Belfiore R, Winslow W \& Oddo S (2017) Necroptosis activation in Alzheimer's disease. Nat. Neurosci. 20, 1236-1246. Available at: http://www.nature.com/doifinder/10.1038/nn.4608.

Cai Z, Jitkaew S, Zhao J, Chiang H-C, Choksi S, Liu J, Ward Y, Wu L \& Liu Z-G (2014) Plasma membrane translocation of trimerized MLKL protein is required for TNF-induced necroptosis. Nat. Cell Biol. 16, 55-65. Available at: http://www.nature.com/articles/ncb2883.

Carvajal FJ, Mira RG, Rovegno M, Minniti AN \& Cerpa W (2018) Age-related NMDA signaling alterations in SOD2 deficient mice. Biochim. Biophys. Acta - Mol. Basis Dis. 1864, 2010-2020. 
Chen X, Li W, Ren J, Huang D, He W, Song Y, Yang C, Li W, Zheng X, Chen P \& Han J (2014) Translocation of mixed lineage kinase domain-like protein to plasma membrane leads to necrotic cell death. Cell Res. 24.

Chen Z, Duan RS, Concha QH, Wu Q, Mix E, Winblad B, Ljunggren HG \& Zhu J (2004) IL-12p35 deficiency alleviates kainic acid-induced hippocampal neurodegeneration in C57BL/6 mice. Neurobiol. Dis. 17, 171-178.

Choi ME, Price DR, Ryter SW \& Choi AMK (2019) Necroptosis: A crucial pathogenic mediator of human disease. JCl Insight 4. Available at: https://pubmed.ncbi.nlm.nih.gov/31391333/ [Accessed May 28, 2021].

Citri A \& Malenka RC (2008) Synaptic plasticity: multiple forms, functions, and mechanisms. Neuropsychopharmacology 33, 18-41. Available at: http://www.ncbi.nlm.nih.gov/pubmed/17728696 [Accessed June 11, 2014].

Daniels BP, Snyder AG, Olsen TM, Orozco S, Oguin TH, Tait SWG, Martinez J, Gale M, Loo Y \& Oberst A (2017) RIPK3 Restricts Viral Pathogenesis via Cell Death-Independent Neuroinflammation. Cell 169, 301-313.e11. Available at: http://dx.doi.org/10.1016/j.cell.2017.03.011.

Debacq-Chainiaux F, Erusalimsky JD, Campisi J \& Toussaint O (2009) Protocols to detect senescence-associated beta-galactosidase (SA- $\beta$ gal) activity, a biomarker of senescent cells in culture and in vivo. Nat. Protoc. 4, 1798-1806. Available at: http://www.nature.com/articles/nprot.2009.191 [Accessed October 15, 2021].

Deepa SS, Unnikrishnan A, Matyi S, Hadad N \& Richardson A (2018) Necroptosis increases with age and is reduced by dietary restriction. Aging Cell 17, 1-5.

Dillon SE, Tsivos D, Knight M, McCann B, Pennington C, Shiel Al, Conway ME, Newson MA, Kauppinen RA \& Coulthard EJ (2017) The impact of ageing reveals distinct roles for human dentate gyrus and CA3 in pattern separation and object recognition memory. Sci. Rep. 7, 1-13. Available at: http://dx.doi.org/10.1038/s41598-017-13853-8.

Duan J, Lv Y Bin, Gao X, Zhou JH, Kraus VB, Zeng Y, Su H \& Shi XM (2020) Association of cognitive impairment and elderly mortality: Differences between two cohorts ascertained 6-years apart in China. Geriatr. 20, 29 Available at: https://bmcgeriatr.biomedcentral.com/articles/10.1186/s12877-020-1424-4 [Accessed April 20, 2021].

Enright AJ (2002) An efficient algorithm for large-scale detection of protein families. Nucleic Acids Res. 30, 1575-1584. Available at: https://pubmed.ncbi.nlm.nih.gov/11917018/ [Accessed 
October 13, 2021].

Finn JT, Weil M, Archer F, Siman R, Srinivasan A \& Raff MC (2000) Evidence that Wallerian degeneration and localized axon degeneration induced by local neurotrophin deprivation do not involve caspases. J. Neurosci. 20, 1333-41. Available at: http://www.ncbi.nlm.nih.gov/pubmed/10662823.

Flurkey K, Currer JM \& Harrison DE (2007) Mouse Models in Aging Research. In The Mouse in Biomedical Research. Elsevier Inc., pp.637-672.

Franceschi C \& Campisi J (2014) Chronic inflammation (Inflammaging) and its potential contribution to age-associated diseases. Journals Gerontol. - Ser. A Biol. Sci. Med. Sci. 69, S4-S9.

Fu Y, Yu Y, Paxinos G, Watson C \& Rusznák Z (2015) Aging-dependent changes in the cellular composition of the mouse brain and spinal cord. Neuroscience $290,406-420$.

Garde E, Mortensen EL, Krabbe K, Rostrup E \& Larsson HBW (2000) Relation between age-related decline in intelligence and cerebral white-matter hyperintensities in healthy octogenarians: $A$ longitudinal study. Lancet 356, 628-634. Available at: https://pubmed.ncbi.nlm.nih.gov/10968435/ [Accessed July 30, 2020].

Geinisman Y, de Toledo-Morrell L, Morrell F, Persina IS \& Rossi M (1992) Age-related loss of axospinous synapses formed by two afferent systems in the rat dentate gyrus as revealed by the unbiased stereological dissector technique. Hippocampus 2, 437-444. Available at: https://pubmed.ncbi.nlm.nih.gov/1308200/ [Accessed April 21, 2021].

Gerdts J, Summers DW, Sasaki Y, DiAntonio A \& Milbrandt J (2013) Sarm1-Mediated Axon Degeneration Requires Both SAM and TIR Interactions. J. Neurosci. 33, 13569-13580. Available at: http://www.ncbi.nlm.nih.gov/pubmed/23946415 [Accessed July 20, 2017].

Gorostieta-Salas E, Moreno-Blas D, Gerónimo-Olvera C, Cisneros B, Court FA \& Castro-Obregón S (2021) Enhanced Activity of Exportin-1/CRM1 in Neurons Contributes to Autophagy Dysfunction and Senescent Features in Old Mouse Brain J. Gebicki, ed. Oxid. Med. Cell. Longev. 2021, 122. Available at: https://www.hindawi.com/journals/omcl/2021/6682336/ [Accessed October 15, 2021].

Hagan C (2017) When are mice considered old? Available at: https://www.jax.org/news-andinsights/jax-blog/2017/november/when-are-mice-considered-old\# [Accessed July 1, 2021].

Hainmueller T \& Bartos M (2020) Dentate gyrus circuits for encoding, retrieval and discrimination of episodic memories. Nat. Rev. Neurosci. 21, 153-168. Available at: /pmc/articles/PMC7115869/ [Accessed July 1, 2021]. 
Hansen M \& Kennedy BK (2016) Does Longer Lifespan Mean Longer Healthspan? Trends Cell Biol. 26, 565-568.

Hernández DE, Salvadores NA, Moya-Alvarado G, Catalán RJ, Bronfman FC \& Court FA (2018) Axonal degeneration induced by glutamate excitotoxicity is mediated by necroptosis. J. Cell Sci. 131, jcs214684. Available at: http://www.ncbi.nlm.nih.gov/pubmed/30333135 [Accessed November 22, 2018].

Hodgson R, Kennedy BK, Masliah E, Scearce-Levie K, Tate B, Venkateswaran A \& Braithwaite SP (2020) Aging: therapeutics for a healthy future. Neurosci. Biobehav. Rev. 108, 453-458.

Hofer M, Hausmann J, Staeheli P \& Pagenstecher A (2004) Cerebral expression of interleukin-12 induces neurological disease via differential pathways and recruits antigen-specific $T$ cells in virus-infected mice. Am. J. Pathol. 165, 949-958. Available at: /pmc/articles/PMC1618590/ [Accessed May 14, 2021].

Holler N, Zaru R, Micheau O, Thome M, Attinger A, Valitutti S, Bodmer JL, Schneider P, Seed B \& Tschopp J (2000) Fas triggers an alternative, caspase-8-independent cell death pathway using the kinase RIP as effector molecule. Nat. Immunol. 1, 489-95. Available at: http://www.nature.com/doifinder/10.1038/82732.

Hussain A, Pooryasin A, Zhang M, Loschek LF, La Fortezza M, Friedrich AB, Blais CM, Üçpunar HK, Yépez VA, Lehmann M, Gompel N, Gagneur J, Sigrist SJ \& Grunwald Kadow IC (2018) Inhibition of oxidative stress in cholinergic projection neurons fully rescues aging-associated olfactory circuit degeneration in drosophila. Elife 7, 1-20.

Hwang I, Oh H, Santo E, Kim D-Y, Chen JW, Bronson RT, Locasale JW, Na Y, Lee J, Reed S, Toth M, Yu WH, Muller FL \& Paik J (2018) FOXO protects against age-progressive axonal degeneration. Aging Cell 17, 1-100. Available at: http://doi.wiley.com/10.1111/acel.12701.

lannielli A, Bido S, Folladori L, Segnali A, Cancellieri C, Maresca A, Massimino L, Rubio A, Morabito G, Caporali L, Tagliavini F, Musumeci O, Gregato G, Bezard E, Carelli V, Tiranti V \& Broccoli V (2018) Pharmacological Inhibition of Necroptosis Protects from Dopaminergic Neuronal Cell Death in Parkinson's Disease Models. Cell Rep. 22, 2066-2079. Available at: http://www.ncbi.nlm.nih.gov/pubmed/29466734 [Accessed February 26, 2018].

Ito Y, Ofengeim D, Najafov A, Das S, Saberi S, Li Y, Hitomi J, Zhu H, Chen H, Mayo L, Geng J, Amin P, DeWitt JP, Mookhtiar AK, Florez M, Ouchida AT, Fan J, Pasparakis M, Kelliher MA, Ravits J \& Yuan J (2016) RIPK1 mediates axonal degeneration by promoting inflammation and necroptosis in ALS. Science 353, 603-8. Available at: http://www.ncbi.nlm.nih.gov/pubmed/27493188. 
Jurk D, Wang C, Miwa S, Maddick M, Korolchuk V, Tsolou A, Gonos ES, Thrasivoulou C, Saffrey MJ, Cameron K \& Zglinicki T von (2012) Postmitotic neurons develop a p21-dependent senescencelike phenotype driven by a DNA damage response. Aging Cell 11, 996. Available at: /pmc/articles/PMC3533793/ [Accessed October 15, 2021].

Kassab R \& Alexandre F (2018) Pattern separation in the hippocampus: distinct circuits under different conditions. Brain Struct. Funct. 223, 2785-2808.

Kataoka K, Matsumoto H, Kaneko H, Notomi S, Takeuchi K, Sweigard JH, Atik A, Murakami Y, Connor KM, Terasaki H, Miller JW \& Vavvas DG (2015) Macrophage- and RIP3-dependent inflammasome activation exacerbates retinal detachment-induced photoreceptor cell death. Cell Death Dis. 6, e1731-10. Available at: http://dx.doi.org/10.1038/cddis.2015.73.

Kennedy BK, Berger SL, Brunet A, Campisi J, Cuervo AM, Epel ES, Franceschi C, Lithgow GJ, Morimoto RI, Pessin JE, Rando TA, Richardson A, Schadt EE, Wyss-Coray T \& Sierra F (2014) Geroscience: Linking aging to chronic disease. Cell 159, 709-713. Available at: http://dx.doi.org/10.1016/j.cell.2014.10.039.

Ko KW, Milbrandt J \& DiAntonio A (2020) SARM1 acts downstream of neuroinflammatory and necroptotic signaling to induce axon degeneration. J. Cell Biol. 219.

Li D, Meng L, Xu T, Su Y, Liu X, Zhang Z \& Wang X (2017) RIPK1-RIPK3-MLKL-dependent necrosis promotes the aging of mouse male reproductive system. Elife 6, 1-21.

Lobo-Silva D, Carriche GM, Castro AG, Roque S \& Saraiva M (2016) Balancing the immune response in the brain: IL-10 and its regulation. J. Neuroinflammation 13, 1-10. Available at: https://jneuroinflammation.biomedcentral.com/articles/10.1186/s12974-016-0763-8 [Accessed March 24, 2021].

López-Otín C, Blasco MA, Partridge L, Serrano M \& Kroemer G (2013) The hallmarks of aging. Cell 153, 1194. Available at: http://dx. [Accessed May 3, 2021].

Luo AL, Yan J, Tang X Le, Zhao YL, Zhou BY \& Li SY (2019) Postoperative cognitive dysfunction in the aged: the collision of neuroinflammaging with perioperative neuroinflammation. Inflammopharmacology 27, 27-37. Available at: https://doi.org/10.1007/s10787-018-00559-0.

Lynch G, Rex CS \& Gall CM (2006) Synaptic plasticity in early aging. Ageing Res. Rev. 5, 255-280.

Marner L, Nyengaard JR, Tang Y \& Pakkenberg B (2003) Marked loss of myelinated nerve fibers in the human brain with age. J. Comp. Neurol. 462, 144-152.

Molnár T, Mázló A, Tslaf V, Szöllősi AG, Emri G \& Koncz G (2019) Current translational potential and underlying molecular mechanisms of necroptosis. Cell Death Dis. 10, 860. Available at: 
https://www.nature.com/articles/s41419-019-2094-z [Accessed November 4, 2021].

Moreno-Blas D, Gorostieta-Salas E, Pommer-Alba A, Muciño-Hernández G, Gerónimo-Olvera C, Maciel-Barón LA, Konigsberg M, Massieu L \& Castro-Obregón S (2019) Cortical neurons develop a senescence-like phenotype promoted by dysfunctional autophagy. Aging (Albany. NY). 11, 6175-6198. Available at: https://www.aging-us.com/article/102181 [Accessed October 15, 2021].

Murphy JM, Czabotar PE, Hildebrand JM, Lucet IS, Zhang JG, Alvarez-Diaz S, Lewis R, Lalaoui N, Metcalf D, Webb Al, Young SN, Varghese LN, Tannahill GM, Hatchell EC, Majewski IJ, Okamoto T, Dobson RCJ, Hilton DJ, Babon JJ, Nicola NA, Strasser A, Silke J \& Alexander WS (2013) The pseudokinase MLKL mediates necroptosis via a molecular switch mechanism. Immunity 39, 443-453. Available at: http://dx.doi.org/10.1016/j.immuni.2013.06.018.

Nadeem M, Spitzbarth I, Haist V, Rohn K, Tauscher K, Rohn K, Bossers A, Langeveld J, PapasavvaStylianou P, Groschup MH, Baumgärtner W, Gerhauser I \& Fast C (2016) Immunolabelling of non-phosphorylated neurofilament indicates damage of spinal cord axons in TSE-infected goats. Vet. Rec. 178, 141.1-141.

Nikoletopoulou V \& Tavernarakis N (2012) Calcium homeostasis in aging neurons. Front. Genet. 0, 200.

O'Shea A, Cohen RA, Porges EC, Nissim NR \& Woods AJ (2016) Cognitive aging and the hippocampus in older adults. Front. Aging Neurosci. 8. Available at: /pmc/articles/PMC5143675/ [Accessed April 20, 2021].

Oñate M, Catenaccio A, Salvadores N, Saquel C, Martinez A, Moreno-Gonzalez I, Gamez N, Soto P, Soto C, Hetz C \& Court FA (2020) The necroptosis machinery mediates axonal degeneration in a model of Parkinson disease. Cell Death Differ. 27, 1169-1185. Available at: https://pubmed.ncbi.nlm.nih.gov/31591470/ [Accessed February 19, 2021].

Orozco S \& Oberst A (2017) RIPK3 in cell death and inflammation: the good, the bad, and the ugly. Immunol. Rev. 277, 102-112. Available at: http://doi.wiley.com/10.1111/imr.12536.

Osterloh JM, Yang J, Rooney TM, Fox AN, Adalbert R, Powell EH, Sheehan AE, Avery MA, Hackett R, Logan MA, MacDonald JM, Ziegenfuss JS, Milde S, Hou Y-J, Nathan C, Ding A, Brown RH, Conforti L, Coleman M, Tessier-Lavigne M, Züchner S, Freeman MR \& Freeman MR (2012) dSarm/Sarm1 is required for activation of an injury-induced axon death pathway. Science 337, 481-4. Available at: http://www.ncbi.nIm.nih.gov/pubmed/22678360 [Accessed July 20, 2017].

Partridge L, Fuentealba M \& Kennedy BK (2020) The quest to slow ageing through drug discovery. Nat. Rev. Drug Discov. 19, 513-532. Available at: https://doi.org/10.1038/ [Accessed July 2, 
2021].

Peters A, Moss MB \& Sethares C (2000) Effects of aging on myelinated nerve fibers in monkey primary visual cortex. J. Comp. Neurol. 419, 364-76. Available at: http://www.ncbi.nlm.nih.gov/pubmed/11016530.

Petzold A (2005) Neurofilament phosphoforms: Surrogate markers for axonal injury, degeneration and loss. In Journal of the Neurological Sciences. Elsevier, pp.183-198. Available at: http://www.jns-journal.com/article/S0022510X05001048/fulltext [Accessed August 3, 2020].

Picon C, Jayaraman A, James R, Beck C, Gallego P, Witte ME, van Horssen J, Mazarakis ND \& Reynolds R (2021) Neuron-specific activation of necroptosis signaling in multiple sclerosis cortical grey matter. Acta Neuropathol. Available at: https://doi.org/10.1007/s00401-021-022747.

Pruitt SC, Freeland A, Rusiniak ME, Kunnev D \& Cady GK (2013) Cdkn1b overexpression in adult mice alters the balance between genome and tissue ageing. Nat. Commun. 4, 2626. Available at: https://www.nature.com/articles/ncomms3626 [Accessed October 18, 2021].

Radhakrishnan H, Stark SM \& Stark CEL (2020) Microstructural Alterations in Hippocampal Subfields Mediate Age-Related Memory Decline in Humans. Front. Aging Neurosci. 12, 1-15.

Rasmussen T, Schliemann T, Sørensen JC, Zimmer J \& West MJ (1996) Memory impaired aged rats: No loss of principal hippocampal and subicular neurons. Neurobiol. Aging 17, 143-147. Available at: https://linkinghub.elsevier.com/retrieve/pii/0197458095020322.

Re DB, Le Verche V, Yu C, Amoroso MW, Politi KA, Phani S, Ikiz B, Hoffmann L, Koolen M, Nagata T, Papadimitriou D, Nagy P, Mitsumoto H, Kariya S, Wichterle H, Henderson CE \& Przedborski $S$ (2014) Necroptosis Drives Motor Neuron Death in Models of Both Sporadic and Familial ALS. Neuron 81, 1001-1008. Available at: http://dx.doi.org/10.1016/j.neuron.2014.01.011.

Rocca WA, Petersen RC, Knopman DS, Hebert LE, Evans DA, Hall KS, Gao S, Unverzagt FW, Langa KM, Larson EB \& White LR (2011) Trends in the incidence and prevalence of Alzheimer's disease, dementia, and cognitive impairment in the United States. Alzheimer's Dement. 7, 8093. Available at: /pmc/articles/PMC3026476/ [Accessed July 1, 2021].

Ropireddy D, Scorcioni R, Lasher B, Buzsáki G \& Ascoli GA (2011) Axonal morphometry of hippocampal pyramidal neurons semi-automatically reconstructed after in vivo labeling in different CA3 locations. Brain Struct. Funct. 216, 1-15. Available at: /pmc/articles/PMC3577356/ [Accessed June 30, 2021].

Rosczyk HA, Sparkman NL \& Johnson RW (2008) Neuroinflammation and cognitive function in aged mice following minor surgery. Exp. Gerontol. 43, 840-846. Available at: 
https://pubmed.ncbi.nlm.nih.gov/18602982/ [Accessed August 11, 2020].

Rosenzweig ES \& Barnes CA (2003) Impact of aging on hippocampal function: Plasticity, network dynamics, and cognition,

Salvadores N \& Court FA (2020) The necroptosis pathway and its role in age-related neurodegenerative diseases: will it open up new therapeutic avenues in the next decade? Expert Opin. Ther. Targets 24, 679-693. Available at: https://www.tandfonline.com/doi/abs/10.1080/14728222.2020.1758668 [Accessed February 23, 2021].

Samson AL, Zhang Y, Geoghegan ND, Gavin XJ, Davies KA, Mlodzianoski MJ, Whitehead LW, Frank D, Garnish SE, Fitzgibbon C, Hempel A, Young SN, Jacobsen A V., Cawthorne W, Petrie EJ, Faux MC, Shield-Artin K, Lalaoui N, Hildebrand JM, Silke J, Rogers KL, Lessene G, Hawkins ED \& Murphy JM (2020) MLKL trafficking and accumulation at the plasma membrane control the kinetics and threshold for necroptosis. Nat. Commun. 11, 3151. Available at: https://www.nature.com/articles/s41467-020-16887-1 [Accessed November 5, 2021].

Santhakumar V, Bender R, Frotscher M, Ross ST, Hollrigel GS, Toth Z \& Soltesz I (2000) Granule cell hyperexcitability in the early post-traumatic rat dentate gyrus: The "irritable mossy cell" hypothesis. J. Physiol. 524, 117-134. Available at: /pmc/articles/PMC2269864/ [Accessed July 1, 2021].

Scharfman HE (2016) The enigmatic mossy cell of the dentate gyrus. Nat. Rev. Neurosci. 17, 562575. Available at: http://www.ncbi.nlm.nih.gov/pubmed/25300886.

Scharfman HE, Smith KL, Goodman JH \& Sollas AL (2001) Survival of dentate hilar mossy cells after pilocarpine-induced seizures and their synchronized burst discharges with area CA3 pyramidal cells. Neuroscience 104, 741-759. Available at: https://pubmed.ncbi.nlm.nih.gov/11440806/ [Accessed July 1, 2021].

Schuitemaker A, van der Doef TF, Boellaard R, van der Flier WM, Yaqub M, Windhorst AD, Barkhof F, Jonker C, Kloet RW, Lammertsma AA, Scheltens P \& van Berckel BNM (2012) Microglial activation in healthy aging. Neurobiol. Aging 33, 1067-1072.

Seehusen F \& Baumgärtner W (2010) Axonal Pathology and Loss Precede Demyelination and Accompany Chronic Lesions in a Spontaneously Occurring Animal Model of Multiple Sclerosis. Brain Pathol. 20, 551-559. Available at: http://doi.wiley.com/10.1111/j.1750-3639.2009.00332.x [Accessed August 3, 2020].

Seo J, Nam YW, Kim S, Oh D-B \& Song J (2021) Necroptosis molecular mechanisms: Recent findings regarding novel necroptosis regulators. Exp. Mol. Med. 2021536 53, 1007-1017. Available at: 
https://www.nature.com/articles/s12276-021-00634-7 [Accessed November 4, 2021].

Shamy JLT, Buonocore MH, Makaron LM, Amaral DG, Barnes CA \& Rapp PR (2006) Hippocampal volume is preserved and fails to predict recognition memory impairment in aged rhesus monkeys (Macaca mulatta). Neurobiol. Aging 27, 1405-1415. Available at: https://pubmed.ncbi.nlm.nih.gov/16183171/ [Accessed April 21, 2021].

Sloviter RS (1987) Decreased hippocampal inhibition and a selective loss of interneurons in experimental epilepsy. Science (80-. ). 235, 73-76. Available at: https://pubmed.ncbi.nlm.nih.gov/2879352/ [Accessed July 1, 2021].

Smith DE, Rapp PR, Mckay HM, Roberts JA \& Tuszynski MH (2004) Memory Impairment in Aged Primates Is Associated with Focal Death of Cortical Neurons and Atrophy of Subcortical Neurons. 24, 4373-4381.

Smith TD, Adams MM, Gallagher M, Morrison JH \& Rapp PR (2000) Circuit-specific alterations in hippocampal synaptophysin immunoreactivity predict spatial learning impairment in aged rats. J. Neurosci. 20, 6587-6593. Available at: https://www.jneurosci.org/content/20/17/6587 [Accessed April 21, 2021].

Spiegel AM, Koh MT, Vogt NM, Rapp PR \& Gallagher M (2013) Hilar interneuron vulnerability distinguishes aged rats with memory impairment. J. Comp. Neurol. 521, 3508-3523.

Stahon KE, Bastian C, Griffith S, Kidd GJ, Brunet S \& Baltan S (2016) Age-Related Changes in Axonal and Mitochondrial Ultrastructure and Function in White Matter. J. Neurosci. 36, 999010001. Available at: http://www.jneurosci.org/cgi/doi/10.1523/JNEUROSCI.1316-16.2016.

Sun L, Wang H, Wang Z, He S, Chen S, Liao D, Wang L, Yan J, Liu W, Lei X \& Wang X (2012) Mixed lineage kinase domain-like protein mediates necrosis signaling downstream of RIP3 kinase. Cell 148, 213-227. Available at: http://dx.doi.org/10.1016/j.cell.2011.11.031.

Tegeler C, O'Sullivan JL, Bucholtz N, Goldeck D, Pawelec G, Steinhagen-Thiessen E \& Demuth I (2016) The inflammatory markers CRP, IL-6, and IL-10 are associated with cognitive functiondata from the Berlin Aging Study II. Neurobiol. Aging 38, 112-117. Available at: https://pubmed.ncbi.nlm.nih.gov/26827649/ [Accessed August 11, 2020].

Theocharidis A, van Dongen S, Enright AJ \& Freeman TC (2009) Network visualization and analysis of gene expression data using BioLayout Express3D. Nat. Protoc. 4, 1535-1550. Available at: https://pubmed.ncbi.nlm.nih.gov/19798086/ [Accessed October 13, 2021].

Thibault O \& Landfield PW (1996) Increase in Single L-Type Calcium Channels in Hippocampal Neurons During Aging. Science (80-. ). 272, 1017-1020. Available at: https://pubmed.ncbi.nlm.nih.gov/8638124/ [Accessed October 18, 2021]. 
Toner CK, Pirogovsky E, Kirwan CB \& Gilbert PE (2009) Visual object pattern separation deficits in nondemented older adults. Learn. Mem. 16, 338-342. Available at: https://pubmed.ncbi.nlm.nih.gov/19403797/ [Accessed April 20, 2021].

Trinchieri G (2003) Interleukin-12 and the regulation of innate resistance and adaptive immunity. Nat. Rev. Immunol. 3, 133-146.

Tsunoda I \& Fujinami RS (2002) Inside-Out versus Outside-In models for virus induced demyelination: axonal damage triggering demyelination. Springer Semin. Immunopathol. 24, 105-25. Available at: https://link.springer.com/article/10.1007/s00281-002-0105-z [Accessed August 3, 2020].

Wang C, Jurk D, Maddick M, Nelson G, Martin-Ruiz C \& Zglinicki T Von (2009) DNA damage response and cellular senescence in tissues of aging mice. Aging Cell 8, 311-323. Available at: https://onlinelibrary.wiley.com/doi/full/10.1111/j.1474-9726.2009.00481.x [Accessed October 18, 2021].

Weber K, Roelandt R, Bruggeman I, Estornes Y \& Vandenabeele P (2018) Nuclear RIPK3 and MLKL contribute to cytosolic necrosome formation and necroptosis. Commun. Biol. 1, 6. Available at: http://www.nature.com/articles/s42003-017-0007-1.

West MJ (1993) Regionally specific loss of neurons in the aging human hippocampus. Neurobiol. Aging 14, 287-293.

Whitmore A V, Lindsten T, Raff MC \& Thompson CB (2003) The proapoptotic proteins Bax and Bak are not involved in Wallerian degeneration. Cell Death Differ. 10, 260-261. Available at: http://www.nature.com/articles/4401147.

Wiley CD \& Campisi J (2021) The metabolic roots of senescence: mechanisms and opportunities for intervention. Nat. Metab. 2021, 1-12. Available at: https://www.nature.com/articles/s42255-02100483-8 [Accessed October 19, 2021].

Wimmer ME, Hernandez PJ, Blackwell J \& Abel T (2012) Aging impairs hippocampus-dependent long-term memory for object location in mice. Neurobiol. Aging 33, 2220-2224. Available at: http://www.ncbi.nlm.nih.gov/pubmed/19772347.

Wittner L, Henze DA, Záborszky L \& Buzsáki G (2007) Three-dimensional reconstruction of the axon arbor of a CA3 pyramidal cell recorded and filled in vivo. Brain Struct. Funct. 212, 75-83. Available at: https://pubmed.ncbi.nlm.nih.gov/17717699/ [Accessed June 30, 2021].

World Health Organization (2019) Global Health Estimates: Life expectancy and leading causes of death and disability. Available at: https://www.who.int/data/gho/data/themes/mortality-andglobal-health-estimates. 
Wu J, Huang Z, Ren J, Zhang Z, He P, Li Y \& Ma J (2013) Mlkl knockout mice demonstrate the indispensable role of Mlkl in necroptosis. Nat. Publ. Gr. 23, 994-1006. Available at: http://dx.doi.org/10.1038/cr.2013.91.

Wyss-Coray T (2016) Ageing, neurodegeneration and brain rejuvenation. Nature 539, 180-186. Available at: http://www.nature.com/doifinder/10.1038/nature20411.

Yang W, Zhou X \& Ma T (2019) Memory Decline and Behavioral Inflexibility in Aged Mice Are Correlated With Dysregulation of Protein Synthesis Capacity. Front. Aging Neurosci. 11, 246. Available at: www.frontiersin.org [Accessed May 26, 2021].

Yang XS, Yi TL, Zhang S, Xu ZW, Yu ZQ, Sun HT, Yang C, Tu Y \& Cheng SX (2017) Hypoxiainducible factor-1 alpha is involved in RIP-induced necroptosis caused by in vitro and in vivo ischemic brain injury. Sci. Rep. 7, 1-11.

Yassa MA, Lacy JW, Stark SM, Albert MS, Gallagher M \& Stark CEL (2011) Pattern separation deficits associated with increased hippocampal CA3 and dentate gyrus activity in nondemented older adults. Hippocampus 21, 968-979. Available at: https://pubmed.ncbi.nlm.nih.gov/20865732/ [Accessed March 11, 2021].

Yoon S, Bogdanov K, Kovalenko A \& Wallach D (2016) Necroptosis is preceded by nuclear translocation of the signaling proteins that induce it. Cell Death Differ. 23, 253-260. Available at: http://dx.doi.org/10.1038/cdd.2015.92.

Yu X-W, Curlik DM, Oh MM, Yin JC \& Disterhoft JF (2017) CREB overexpression in dorsal CA1 ameliorates long-term memory deficits in aged rats. Elife 6. Available at: /pmc/articles/PMC5214885/ [Accessed October 18, 2021].

Yuan A, Rao M V., Veeranna \& Nixon RA (2012) Neurofilaments at a glance. J. Cell Sci. 125, 32573263.

Zia A, Pourbagher-Shahri AM, Farkhondeh T \& Samarghandian S (2021) Molecular and cellular pathways contributing to brain aging. Behav. Brain Funct. 2021171 17, 1-30. Available at: https://behavioralandbrainfunctions.biomedcentral.com/articles/10.1186/s12993-021-00179-9 [Accessed October 18, 2021]. 

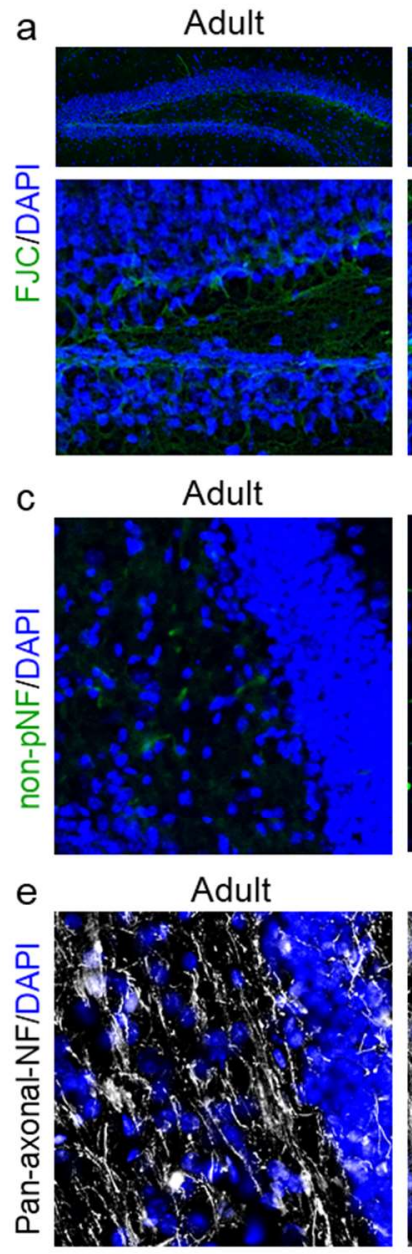

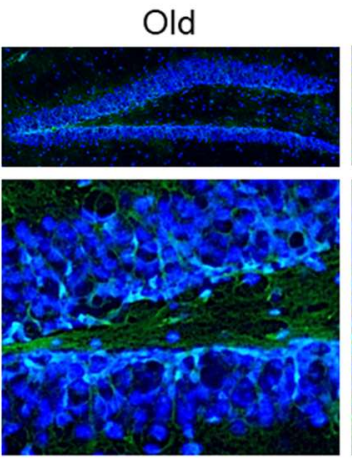

Old

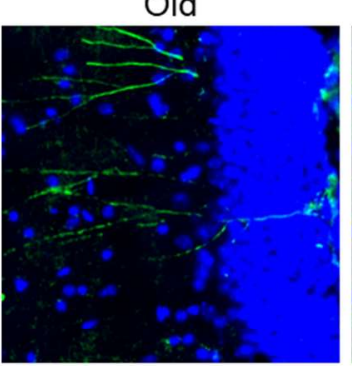

Old

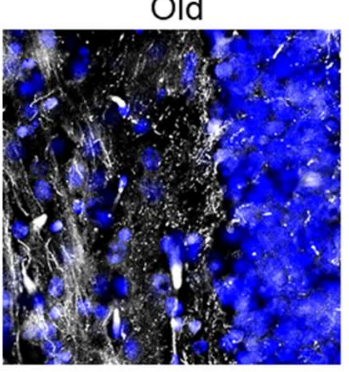

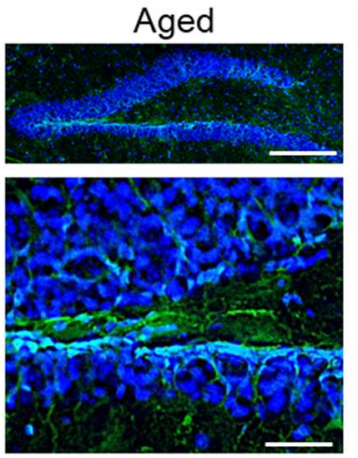
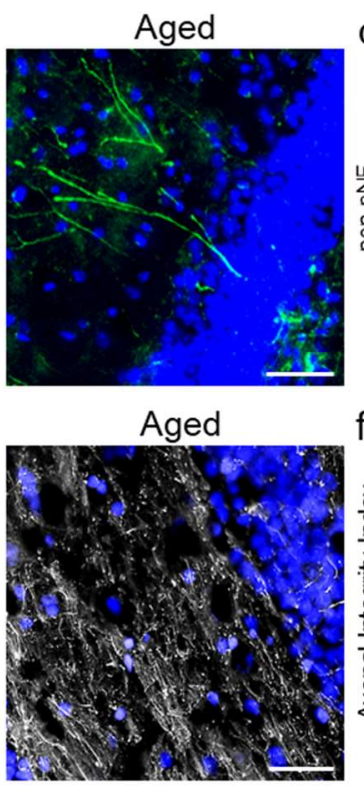

d
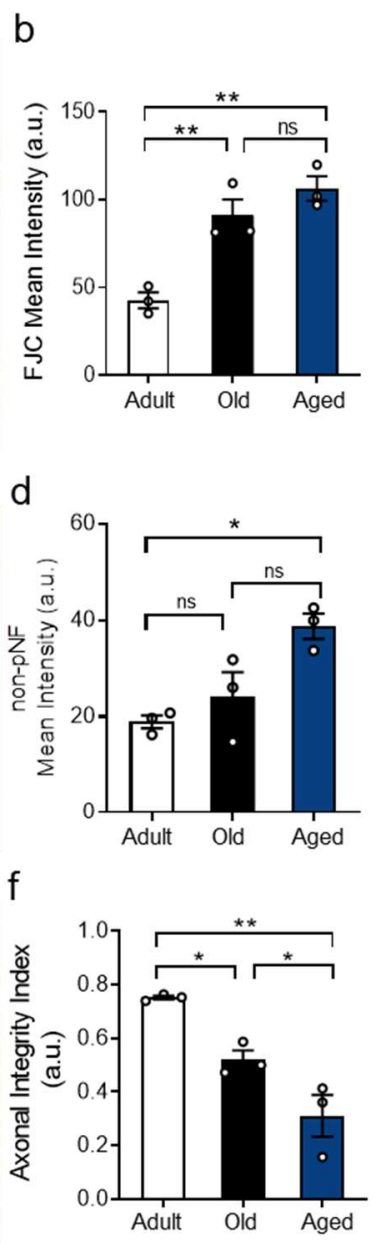

Fig 1. Increased axonal degeneration in the hippocampus during aging. Representative images of hippocampal sections from adult (3-6 month), old (12-25 month) and aged mice (more than 24 month) stained with Fluoro Jade C (FJC, green), non-phosphorylated neurofilament (non-pNF) and pan-axonal NF (a,c,e). Nuclear staining with DAPI (blue) was used to identify the granular layer. (b,d) The mean fluorescence intensity was evaluated in FJC and non-pNF staining to determine levels of neurodegeneration in the hilus according to age. (f) Axonal degeneration was assessed in the DG hilus using the axonal integrity index in hippocampal sections immunostained with the pan-axonal NF antibody. Calibration bars, $250 \mu \mathrm{m}$ (entire DG) and $50 \mu \mathrm{m}$ for magnified hippocampal images. Values are the result of the analysis of $n=3-4$ mice per group. One-way ANOVA with Tukey analysis for multiple comparisons, ${ }^{*} p:<0.05 ;{ }^{* *} p<0.01 ;{ }^{* * *} p<0.005 ;{ }^{* * * *} p<0.001$. 
Fig. 2
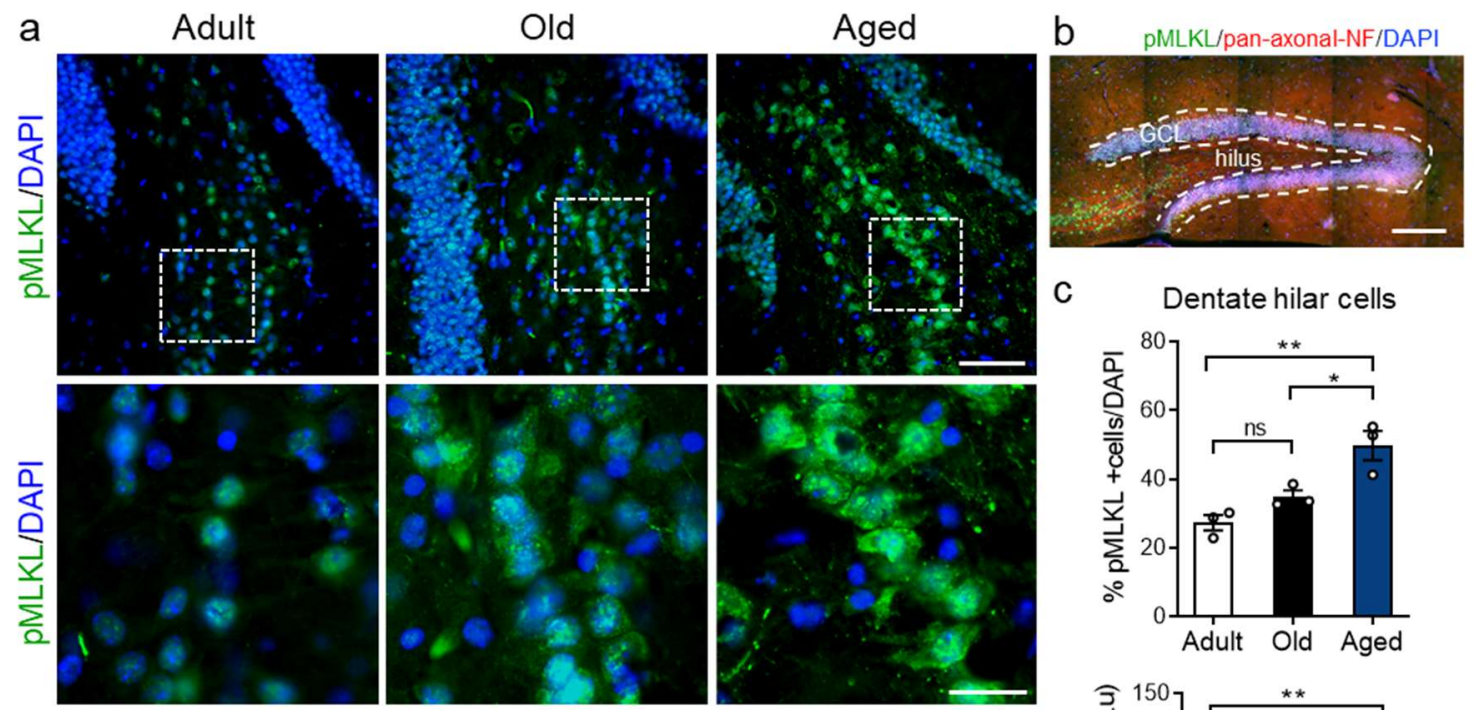

C

Dentate hilar cells
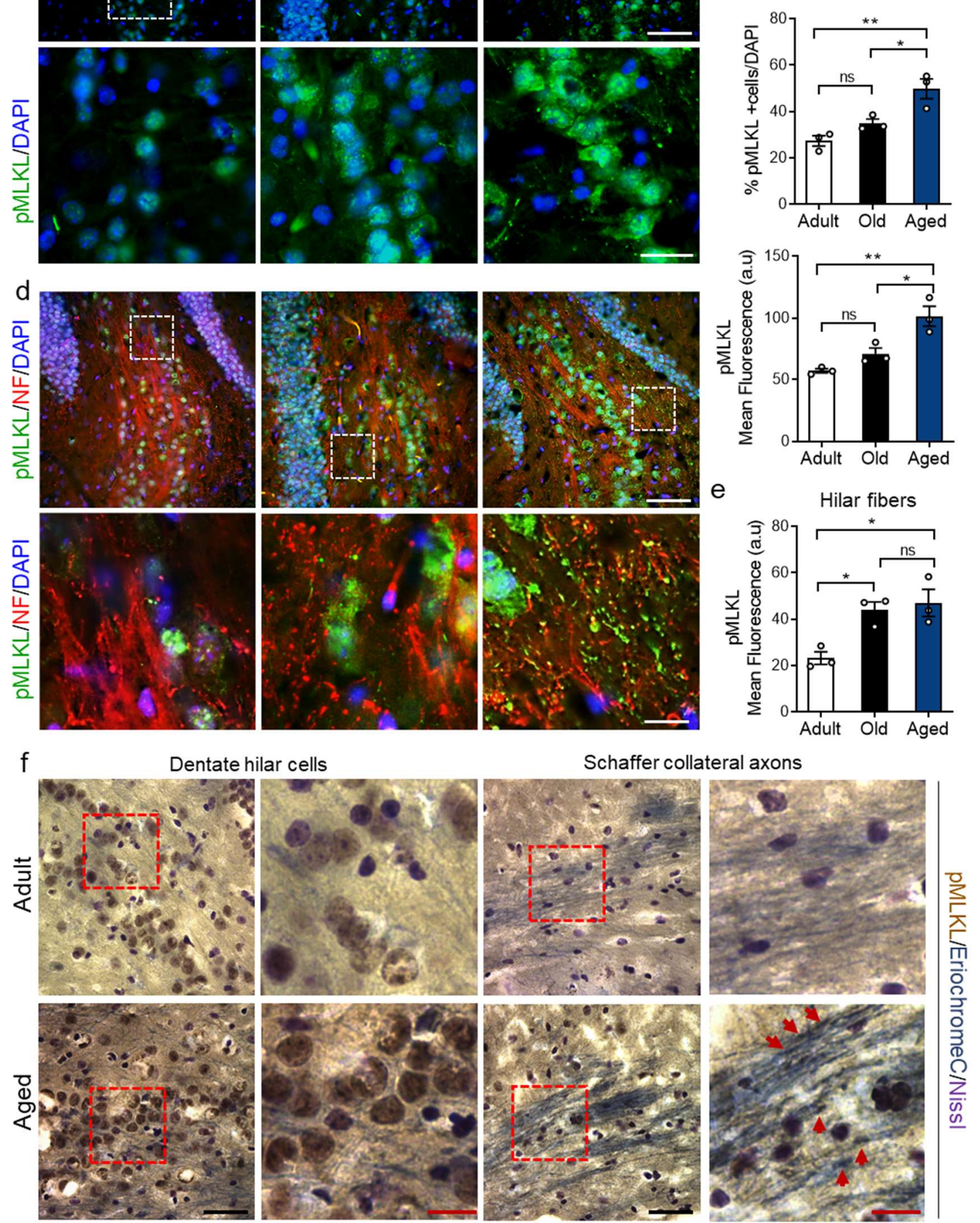
Fig 2. Progressive increase of phosphorylated MLKL levels in the hippocampal hilus along aging. (a) MLKL phosphorylation at serine 345 (green) was evaluated in adult (3-6 month), old (1225 month) and aged mice (more than 24 month) (bar=25 $\mu \mathrm{m}$ and $10 \mu \mathrm{m}$ for magnification). (b) Mosaic reconstruction of the hippocampal dentate gyrus (DG) illustrates each subfield: the granular cell layer (GCL), and the hilus, showing pMLKL in green (bar=100 $\mu \mathrm{m}$ ). (c) Quantification of pMLKL signal in hilar cells is plotted as the percentage of pMLKL positive cells normalized against the total cell number in the hilar area (upper graph), and as pMLKL mean intensity (lower graph). (d) Axonal pMLKL was detected in NF-positive axons in the hilus, dotted boxes illustrate magnified areas shown in the bottom panel. Calibration bars, $25 \mu \mathrm{m}$ and $5 \mu \mathrm{m}$ for inset. (e) pMLKL mean intensity was analyzed in hilar fibers along aging. Error bars, mean \pm SEM, ${ }^{*} p:<0.05 ;{ }^{* *} p<0.01$. Statistical significance was determined by one-way ANOVA with Tukey analysis for multiple comparisons. (f) Immunohistochemistry against pMLKL (brown) and Eriochrome-C staining (myelinated axons, blue). Calibration bar $40 \mu \mathrm{m}$. Red dotted boxes showed image magnification of each condition. Calibration bar, $15 \mu \mathrm{m}$. Necroptosis activation in DG axons and in Schaffer collateral projections of the hippocampus are shown with red arrowheads. Increased pMLKL was also observed in neuronal somas (dark brown/+Nissl, violet). 
Fig. 3

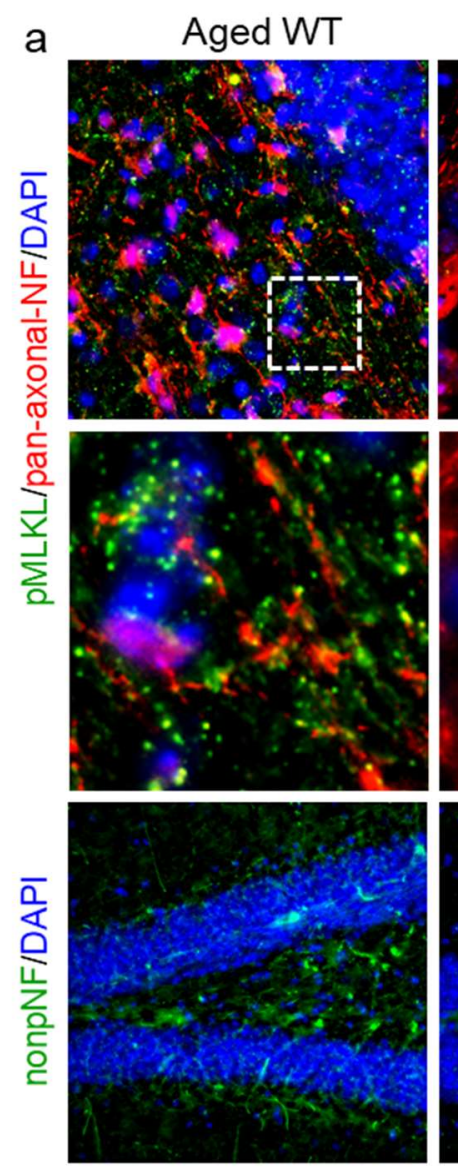

Aged Mlkl-KO
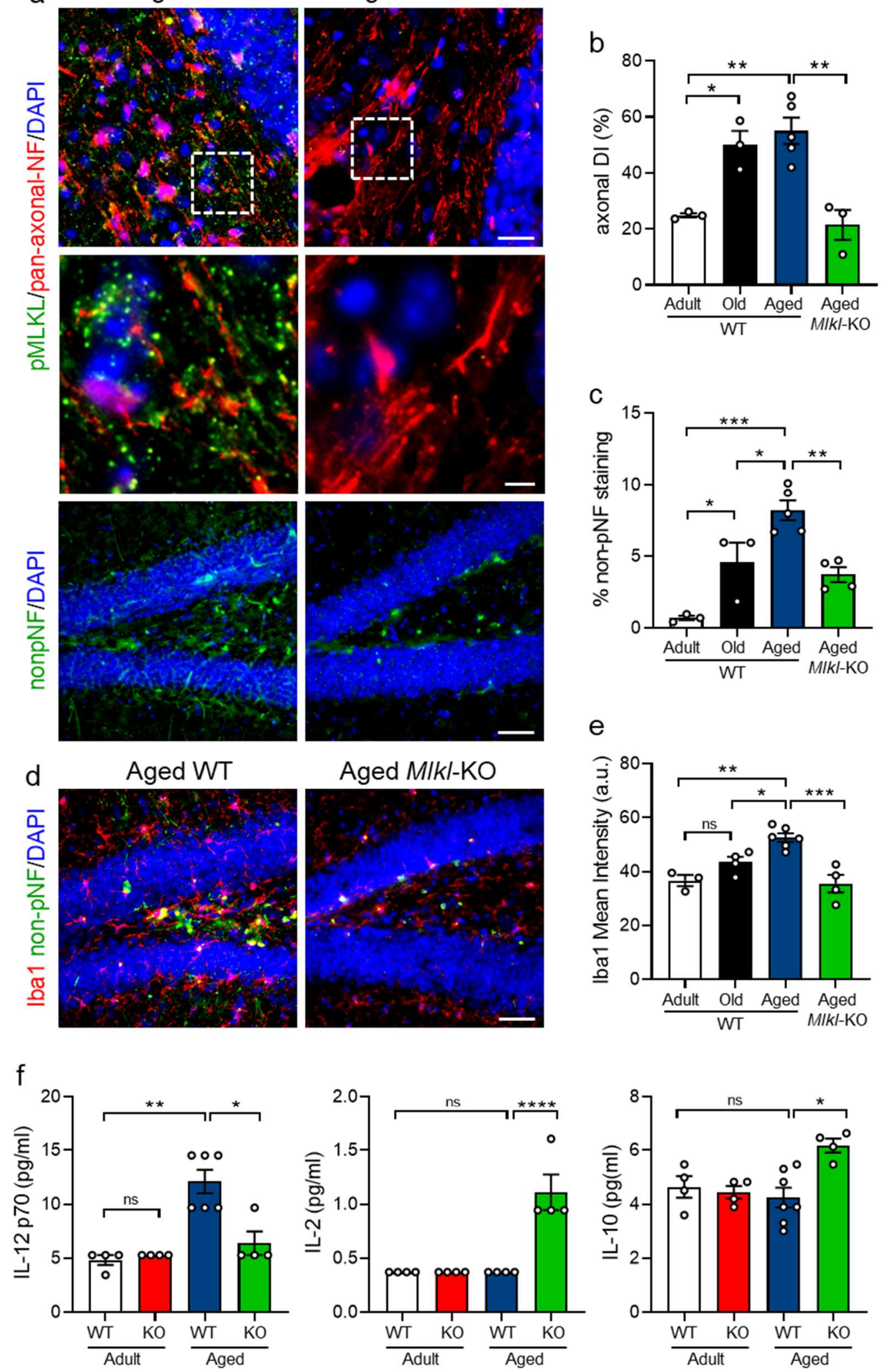
Fig 3. MIkl deficiency protects the brain from age-associated axonal degeneration and neuroinflammation. Axonal degeneration and neuroinflammation were evaluated in the DG hilus of aged WT and Mlkl-KO mice and compared with the values of the normal aging curve ( $N=3-5$ mice per group). (a) Axonal degeneration was detected by immunostaining of axons with the pan-axonalNF marker (red) and evaluated by calculating the axonal degeneration index (DI). Calibration bars, $25 \mu \mathrm{m}$ and $5 \mu \mathrm{m}$ for insets. (b) Increased non-pNF signal was also used as a positive marker of axonal degeneration. Calibration, bar, $50 \mu \mathrm{m}$. (c) Quantification of non-pNF signal plotted as the percentage of stained area in the hilus. (d) Microglia activation was measured by lba1 immunodetection. Calibration bar, $50 \mu \mathrm{m}$. (e) Microglia activation quantified through lba1 mean intensity. (f) Cytokines levels in the hippocampus of adult versus aged WT and MIKl-KO mice were assessed by Luminex High Performance Assay. Plots represent absolute cytokine levels (pg/ml), including IL-12, IL-2 and IL-10 in aged WT and Mlkl-KO hippocampus, ( $\mathrm{N}=4-7$ mice per group). Error bars, mean $\pm \mathrm{SEM}$, ${ }^{*} p:<0.05 ;{ }^{* *} p<0.01 ;{ }^{* *} p<0.005 ;{ }^{* * *} p<0.001$. Statistical significance was determined by one-way ANOVA with Tukey analysis for multiple comparisons. 
Fig. 4

a

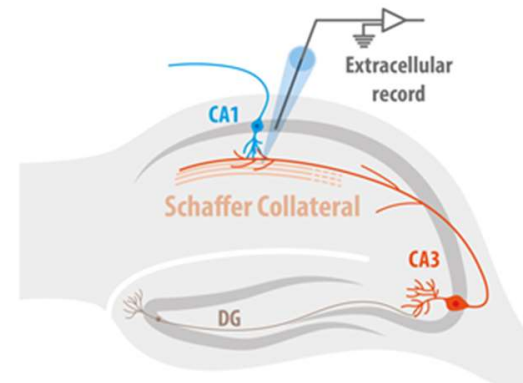

C

e

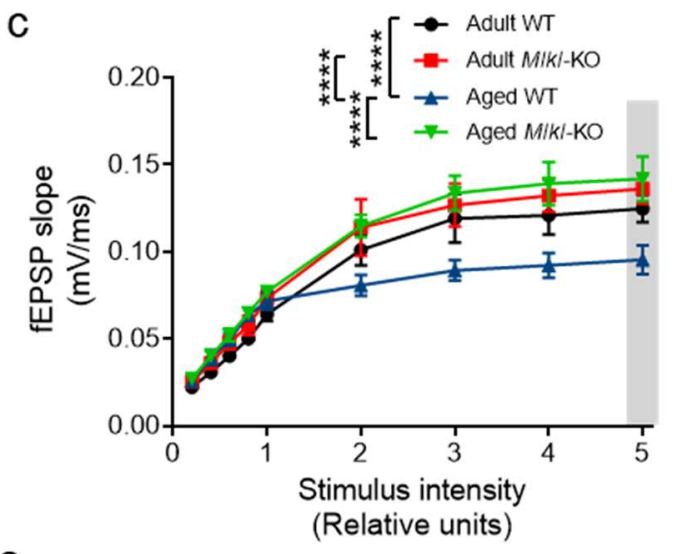

b

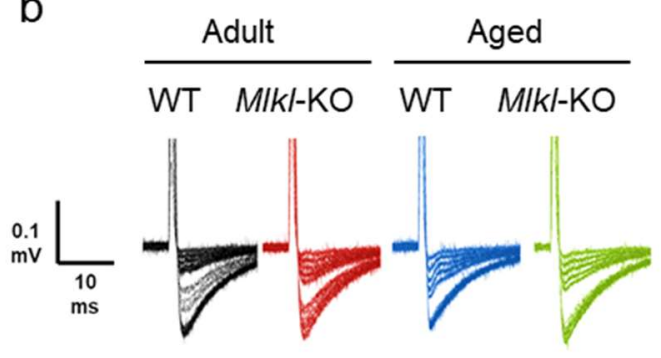

d

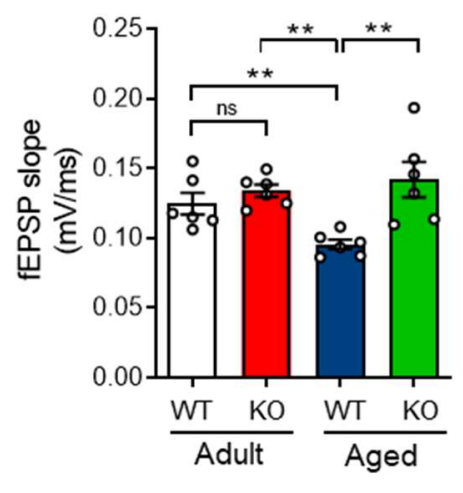

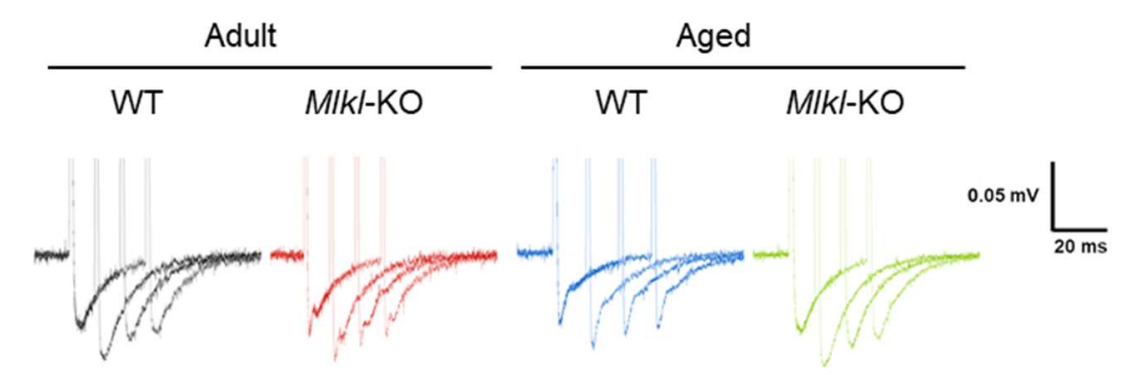

f

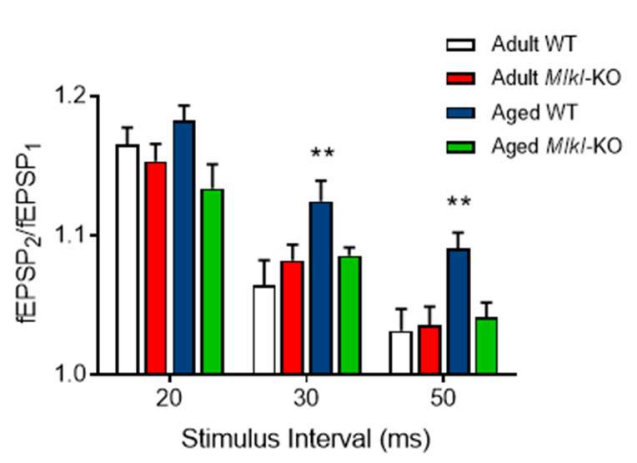

h
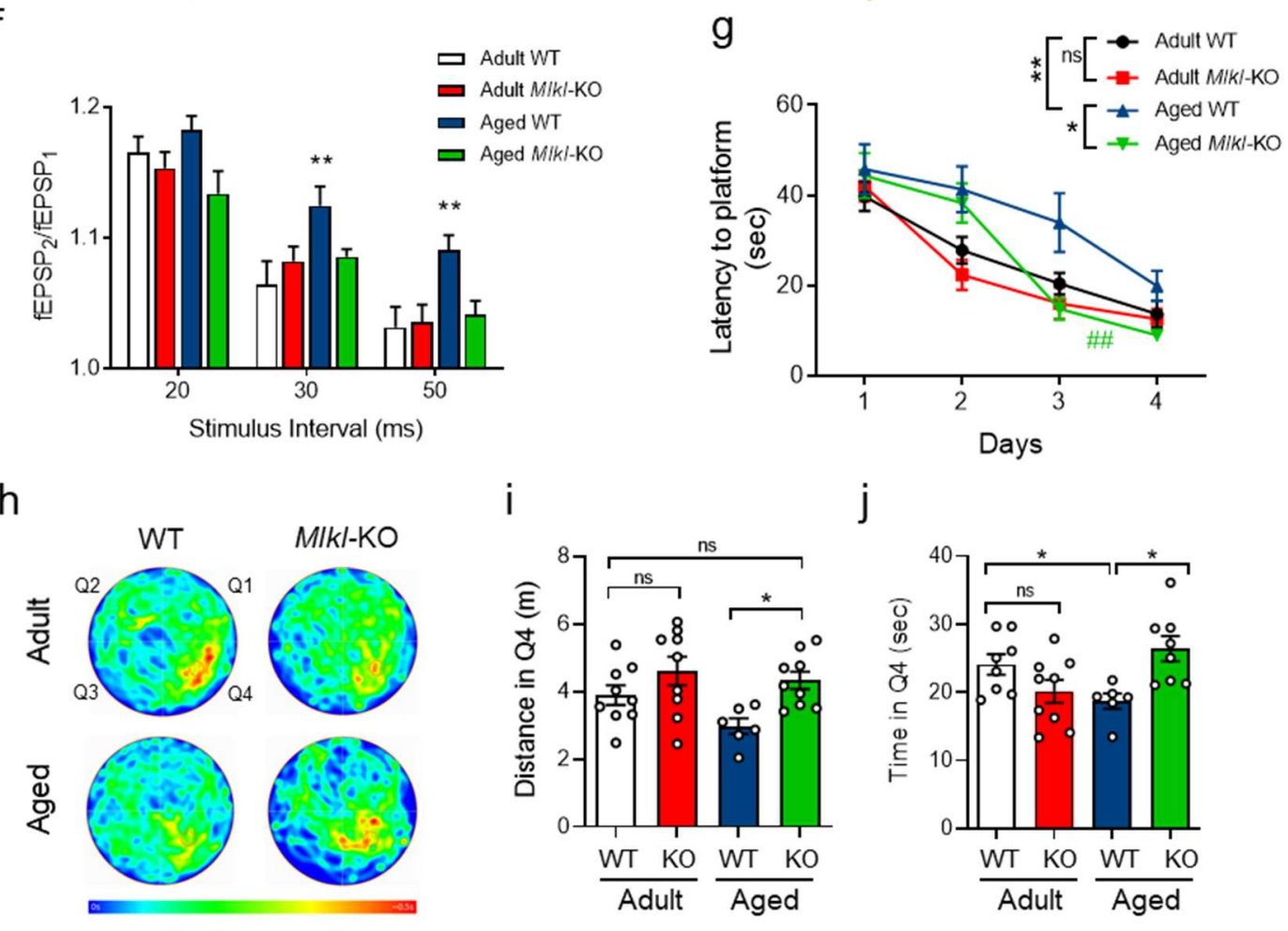

i
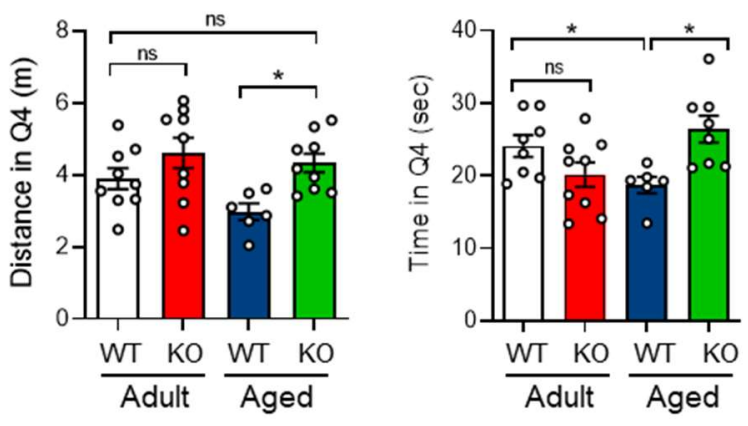
Figure 4. Hippocampal function and memory are improved in aged MIkl-KO mice. (a) Field excitatory post synaptic potential (fEPSP) was measured in hippocampal slices in the CA3-CA1 circuit as described in the scheme. $(\mathbf{b}, \mathbf{c})$ Average traces of the evoked potentials recorded in each condition were used to plot the fEPSP slope upon several stimulus intensity. (d) The average fEPSP slope at the highest stimulus (gray bar in c) was plotted as bar graphs, showing significant differences between groups. $(\mathbf{e}, \mathbf{f})$ Paired pulse facilitation was measured as the facilitation index (fEPSP $\left./ \mathrm{fEPSP}_{1}\right)$. Values are the result of the analysis of $\mathrm{N}=3$ mice per group ( $n=3$ slices per mice). Error bars, mean $\pm S E M$, ${ }^{* *} p<0.01$. Statistical significance was determined by one-way ANOVA. Hippocampal-dependent behavioral function was evaluated using the standard Morris water maze (MWM) navigation task. (g) Escape latency was measured as the time to reach the hidden platform during the learning curve in aged and adult WT vs MIkl-KO mice. (h) Mean heatmaps specify location each mice cohort along time in pseudo-color during memory testing (day 5). The quadrant Q4 designates the initial location of the hidden platform during training. (i,j) Travelled distance and time spent in Q4, respectively. Values are the result of the analysis of $n=7-10$ mice per group. One-way ANOVA with Tukey correction for multiple comparison, ${ }^{*} p:<0.01$; Two-way ANOVA plus Sidak's multiple comparison test was applied for the learning curve data analysis. Significance between curves was determined with $a{ }^{*} p=0.0209$ for aged WT and Mlkl-KO comparison, and ${ }^{* *} p=0.0015$ for differences between adult and aged WT mice. Differences observed at day 3 between aged WT vs MIkl-KO correspond to $\# p=0.0043$. 
Fig. 5

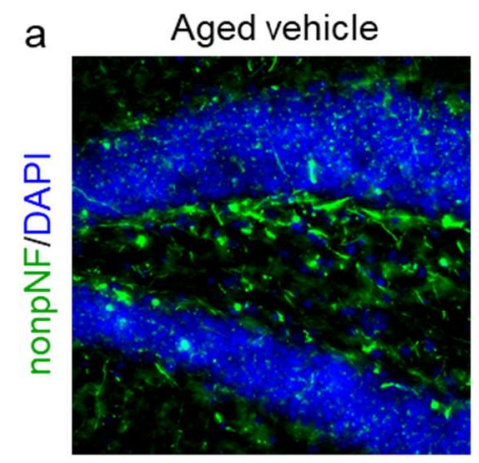

C

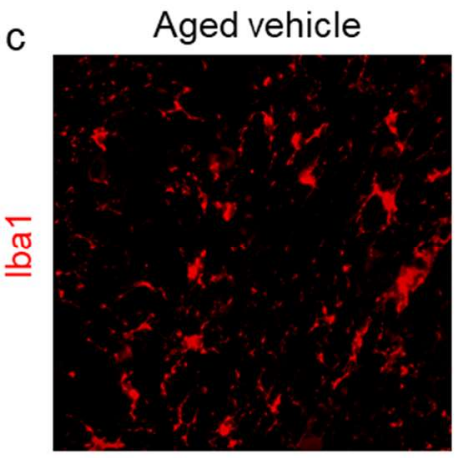

e

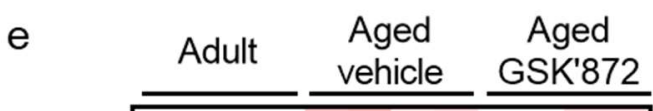

Aged GSK'872

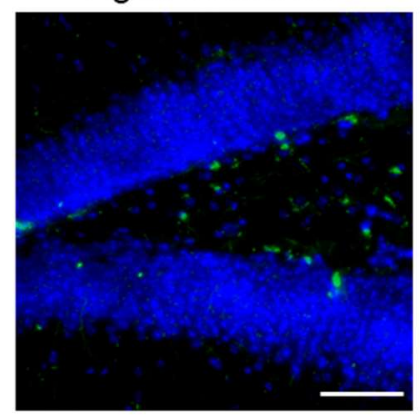

Aged GSK'872

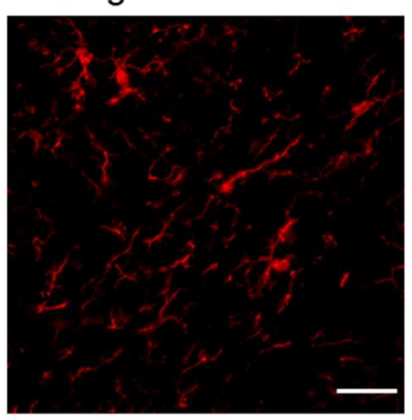

b

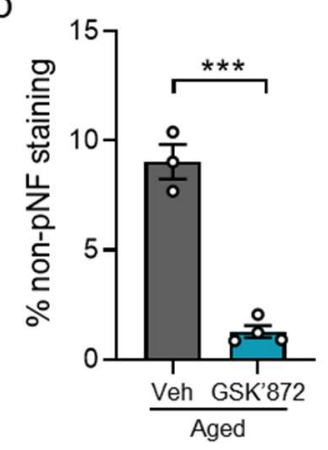

d

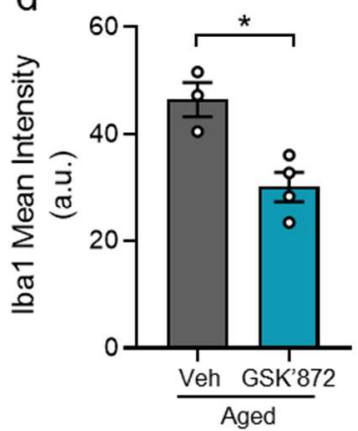

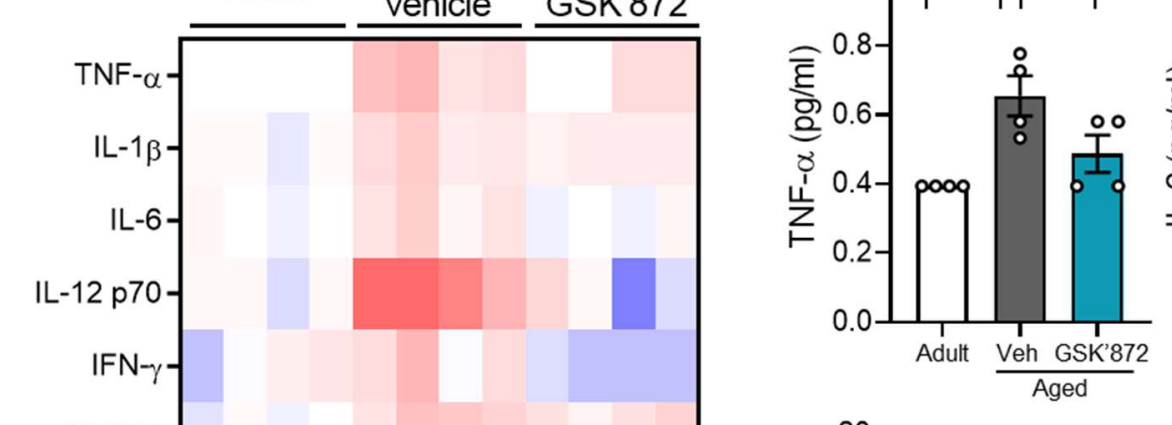
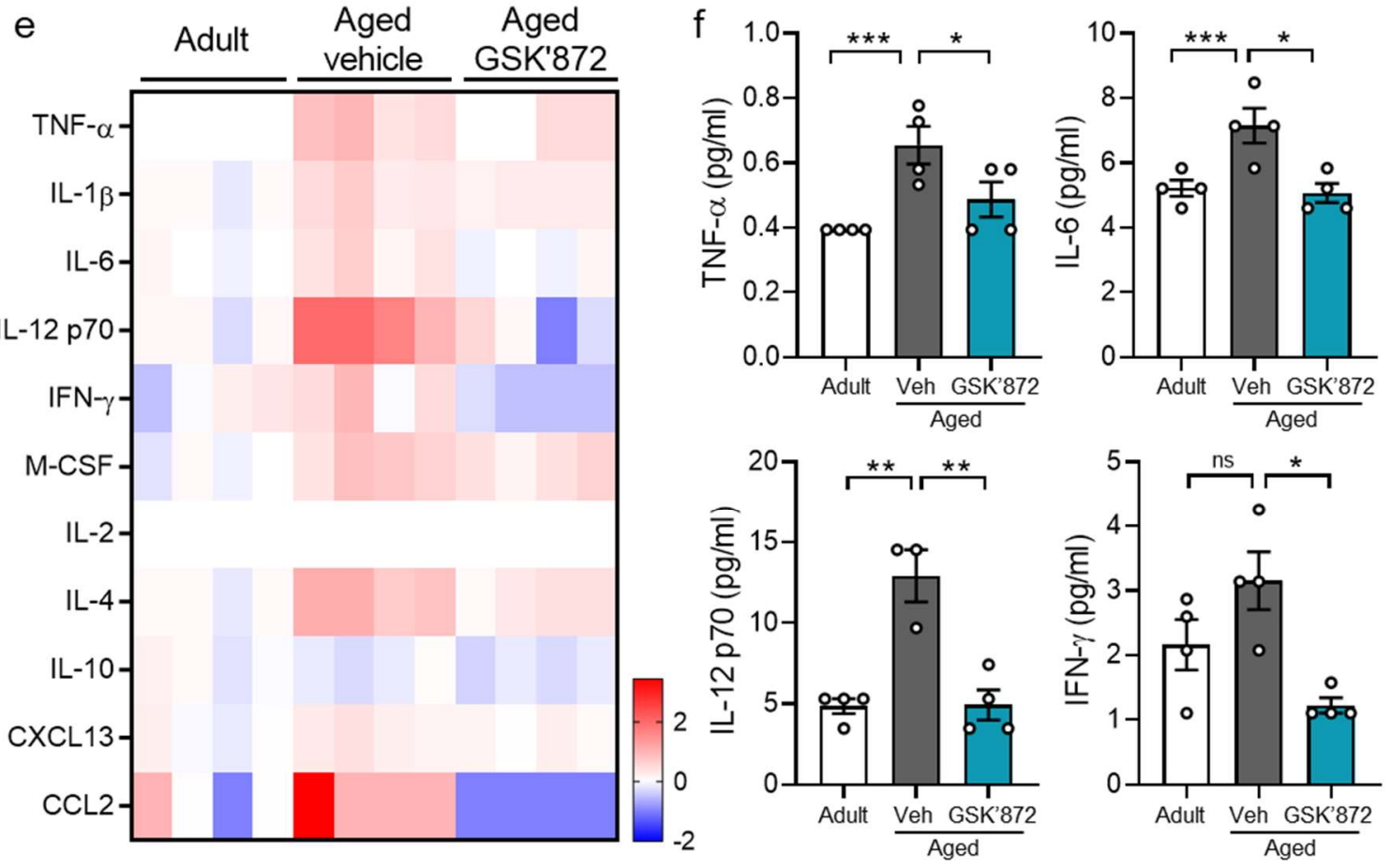
Figure 5. Short-term and systemic RIPK3 inhibition reverts age-associated axonal degeneration and neuroinflammation. Aged mice (23-month-old) treated with vehicle or GSK'872, intraperitoneally delivered through an osmotic pump, diffusion rate $=0.11 \mu \mathrm{l} / \mathrm{hr} / 28$ days. (a,b) Axonal degeneration detected by non-pNF (calibration bar, $50 \mu \mathrm{m}$ ) was quantified and plotted as the percentage of stained area in the DG hilus. (c,d) Microglia activation assessed by lba1 immunodetection (calibration bar, 50 $\mu \mathrm{m}$ ) and quantified as Iba1 mean intensity in the hilus. (e) The heatmap shows fold change levels of cytokines and chemokines analyzed in the hippocampus of adult versus aged-vehicle and aged-GSK' 872 treated mice ( $n=4$, separated in each column). (f) Absolute levels (pg/ml) of TNF- $\alpha$, IL-6, IL-12p70 and IFN- $\gamma$ in the hippocampus of adult (white bar), aged-vehicle (gray) and aged-GSK'872 (light blue) treated mice. ( $\mathrm{N}=3-4$ mice per group). Error bars, mean \pm SEM, ${ }^{*} p:<0.05 ;{ }^{* *} p<0.01 ;{ }^{* * *} p<0.005$. Statistical significance was determined by one-way ANOVA with Tukey analysis for multiple comparisons. 
a

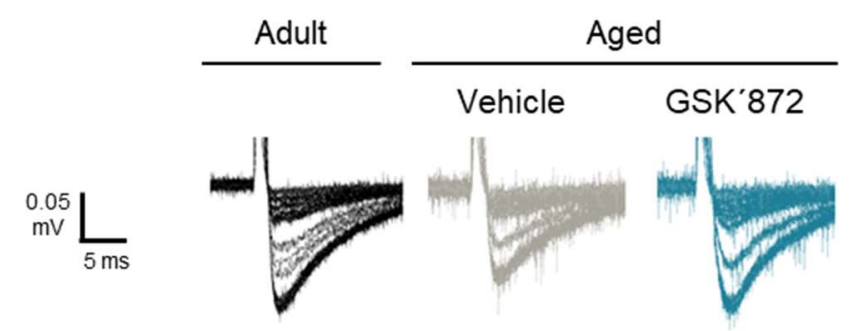

b

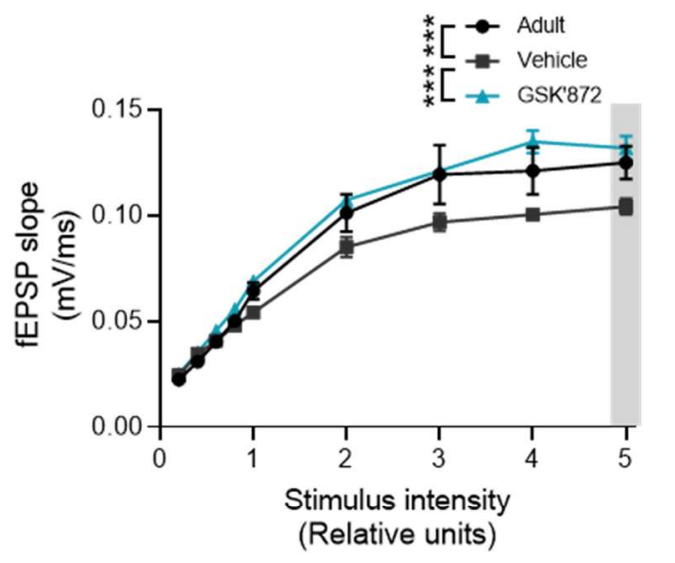

C

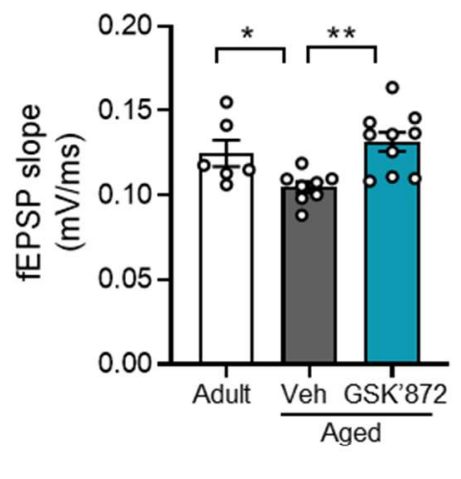

d

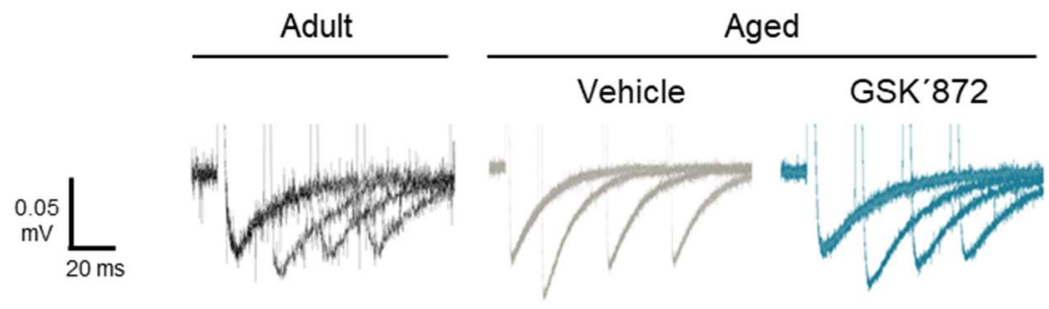

e

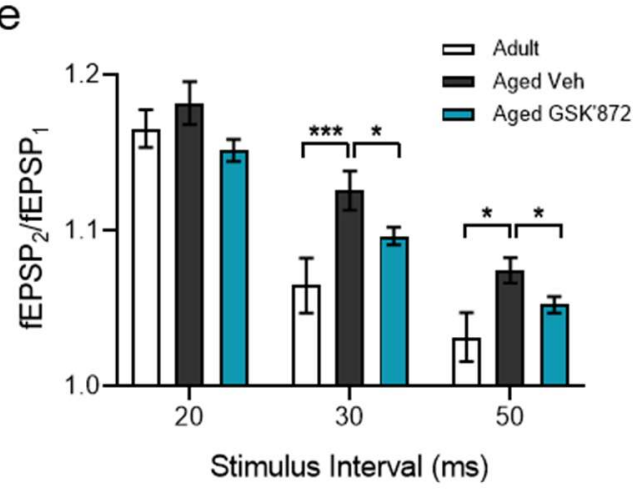

Fig. 6

g

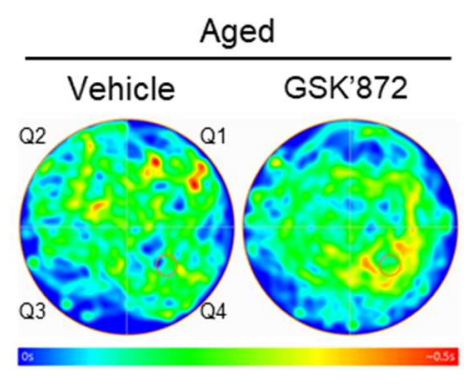

f

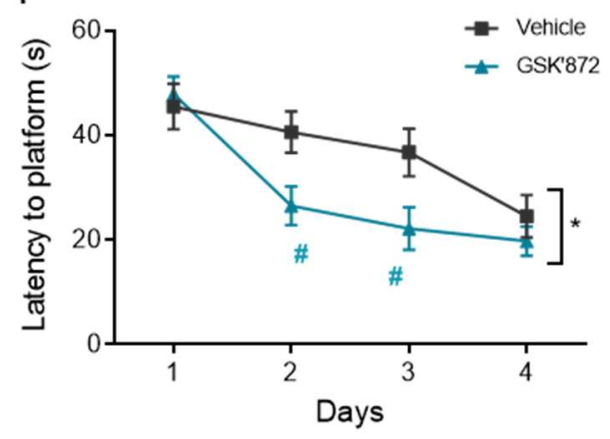

$\mathrm{h}$

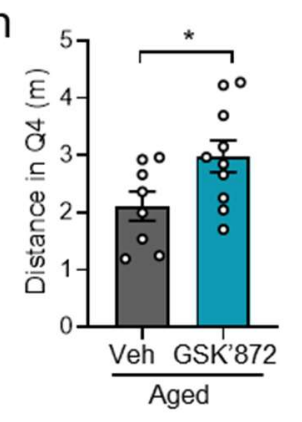

i

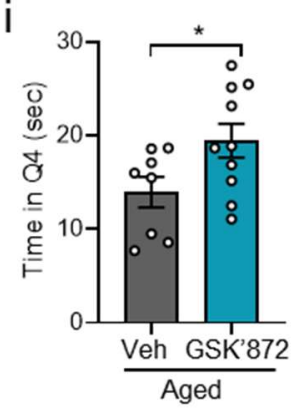


Figure 6. Pharmacological necroptosis inhibition restores hippocampal function and memory in aged mice. (a) Average traces of the evoked potentials recorded in each condition were used to plot the fEPSP slope upon several stimulus intensity in b. (c) The average fEPSP slope at the highest stimulus (gray bar in a) was plotted. Significant differences were detected between groups. (d,e) Paired pulse facilitation was measured as the facilitation index (fEPSP $/ \mathrm{fEPSP}_{1}$ ). Values are the result of the analysis of $\mathrm{N}=3$ mice per group ( $n=3$ slices per mice). Error bars, mean $\pm S E M,{ }^{* *} p<0.01$. Statistical significance was determined by one-way ANOVA. Hippocampal-dependent memory was evaluated with the MWM test. (f) Escape latency of vehicle-treated vs GSK'872-treated aged mice measured during the learning curve. (g) The swimming heatmaps indicating the average mice location along time (pseudo-color) during memory testing (day 5) show increased preference of aged mice treated with GSK'872 to the original location of the platform (Q4) compared with aged-vehicle mice. (h, i) Travelled distance and the time spent in the target quadrant Q4 were plotted. Values are the result of the analysis of $n=8-10$ mice. T-test with Welch's correction, ${ }^{*} p:<0.01$. Two-way ANOVA with Sidak's multiple comparison test was applied for the learning curve data. Significance between both curves was determined with a * $p=0.0259$. Differences between vehicle vs GSK' 872 at day 2 and 3 correspond to ${ }^{p}=0.0210$ and $p=0.0328$, respectively. 
Fig. 7

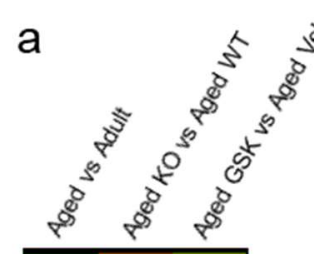

$b$

C

Aged vs Adult

CAged KO vs Aged WT

CAged GSK vs Aged Veh

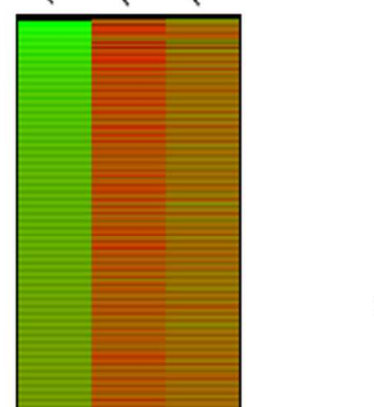

Synaptogenesis--
CREB Signaling in Neurons-
Synaptic LTD--
Synaptic LTP--
NGF Signaling-
Neurotrophin/TRK Signaling-

Synaptic Mechanisms
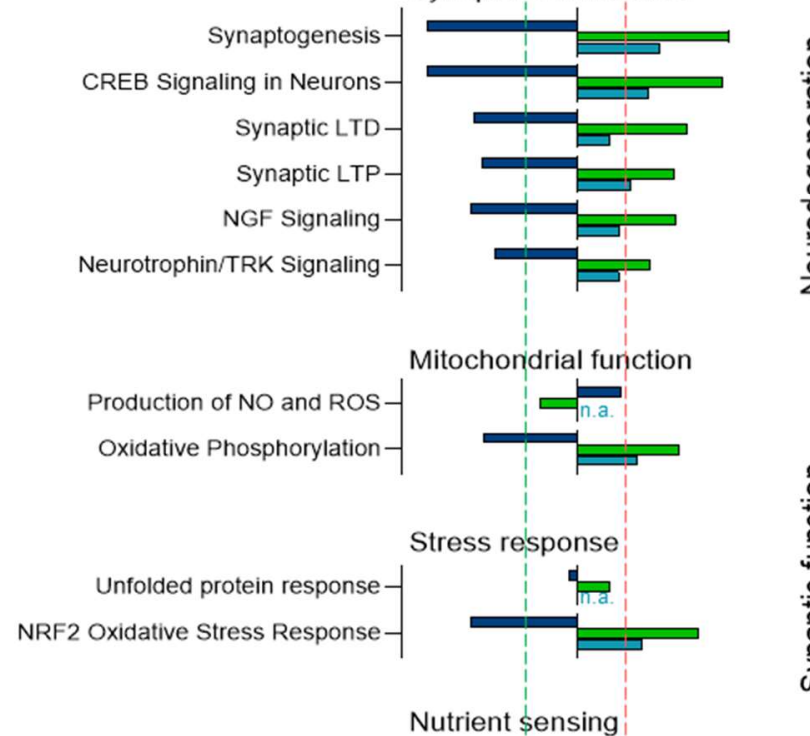

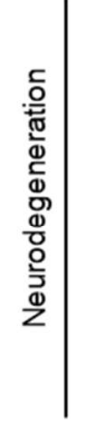

Cellular homeostasis

Neuronal density

Neuronal cell death

Amyloidosis

Apoptosis of neurons

Neurodegeneration -

Necrosis

Apoptosis
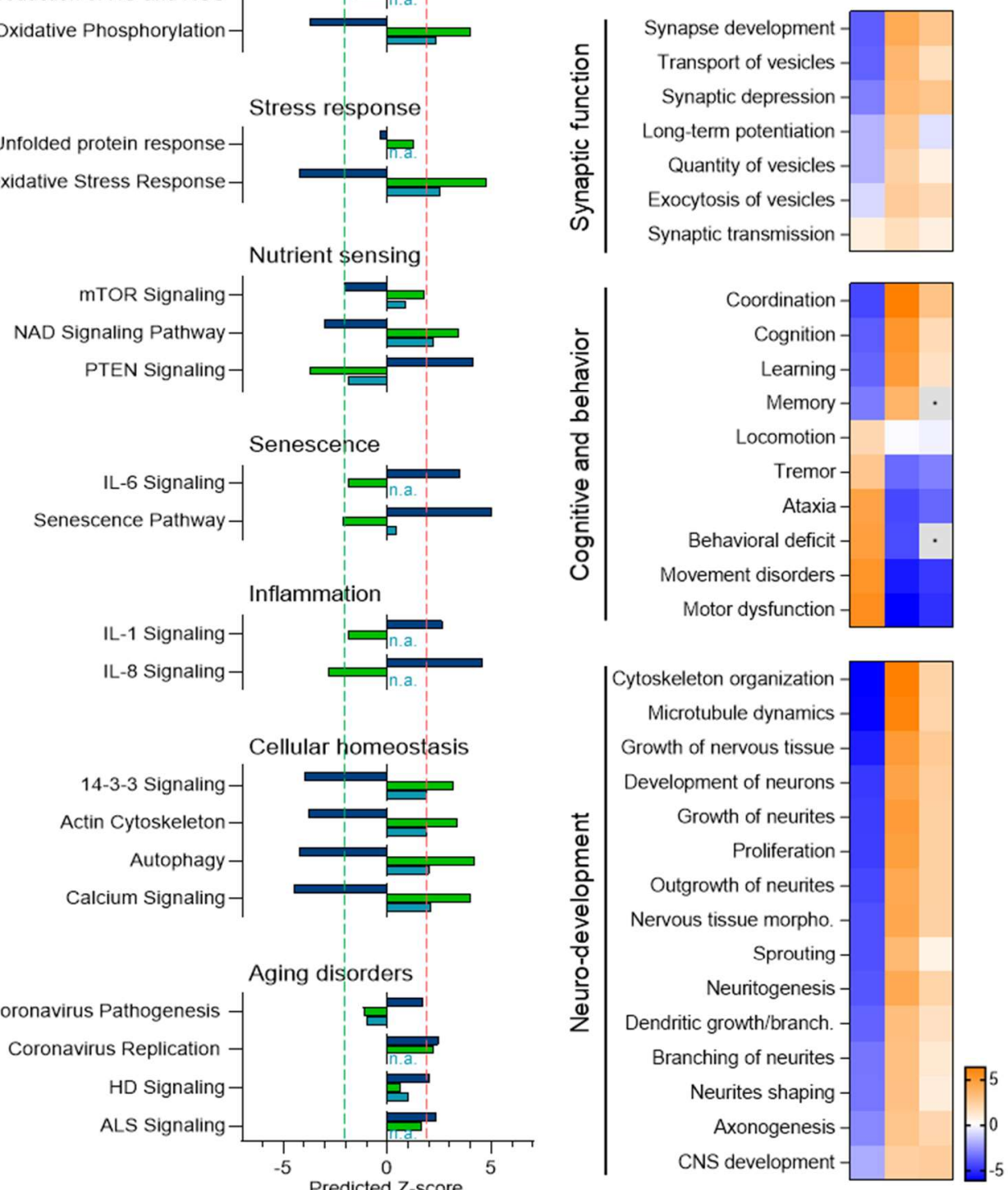
Figure 7. Proteomic analysis reveals opposing directionality in protein expression and agingassociated biofunctions between normal aging and necroptosis-targeted inhibition. (a) Heat map illustrating proteins exhibiting opposing directionality in expression between "normal" aging (aged vs. adult) and necroptosis-inhibited processes in aged mice (MIkl-KO and GSK'872), as identified through unbiased expression profile clustering (Fig $\mathbf{S 8}$ and Fig S9). Heat map depicts mean expression ratio $(\log 2)$ of $n=4$ mice per experimental group versus their respective controls. Red represents upregulation compared to control within each respective analysis, while green represents downregulation. (b) Selection of canonical pathways classified as aging hallmarks (Supplementary Fig S10) were identified through comparative analyses of proteins correlating with necroptosistargeted inhibition of aging phenotypes. Opposing directionality was observed in predicted activation or inhibition scores between "normal" aging cohort, and both MIkl-KO or GSK'872-treated mice. A predicted $z$-score $>2$ or $<-2$ (indicated by dotted lines) is considered statistically significant. For description of statistical tests utilized to generate predictive z-score see Methods. (c) Selected diseases and biofunctions implicated in brain rejuvenation and derived through comparative analyses of proteins correlating with necroptosis-targeted inhibition of aging phenotypes. Heatmaps shows the opposing directionality in predicted activation or inhibition scores between "normal" aging, and both MIkI-KO or GSK'872-treated mice. For description of statistical tests utilized to generate predictive zscores, see Methods. 
Fig. 8

a Aged vs Adult

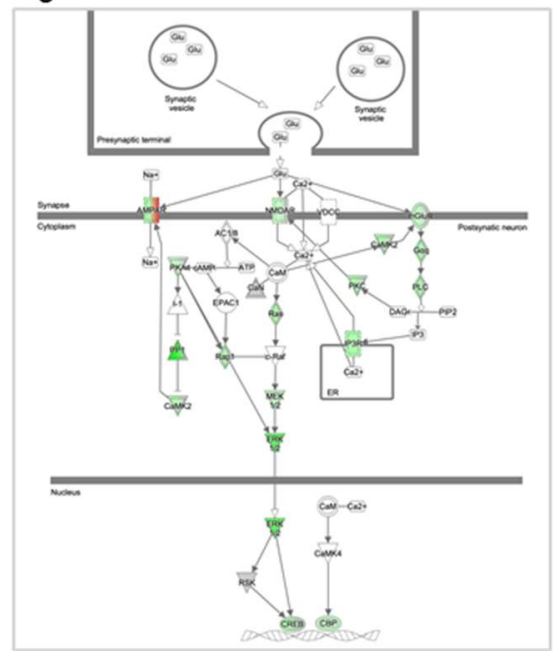

b synaptic LTP signaling

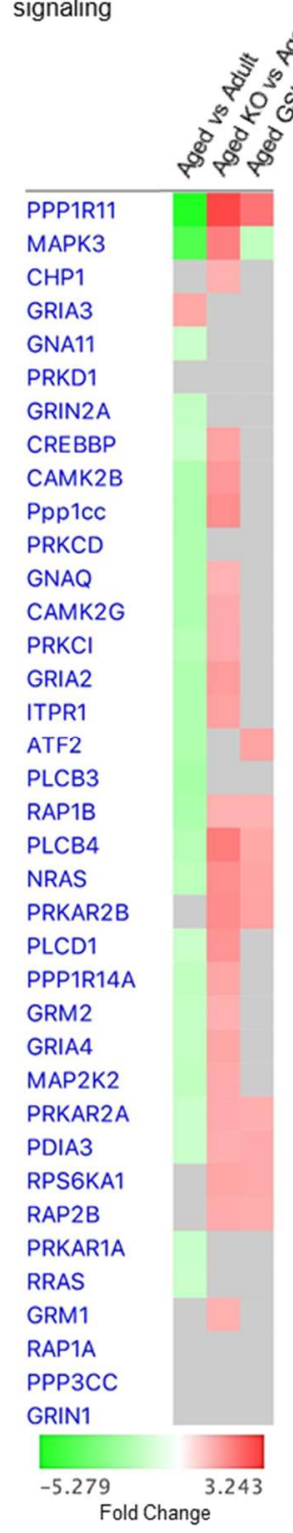

d
C

Aged MIKl-KO vs Aged WT

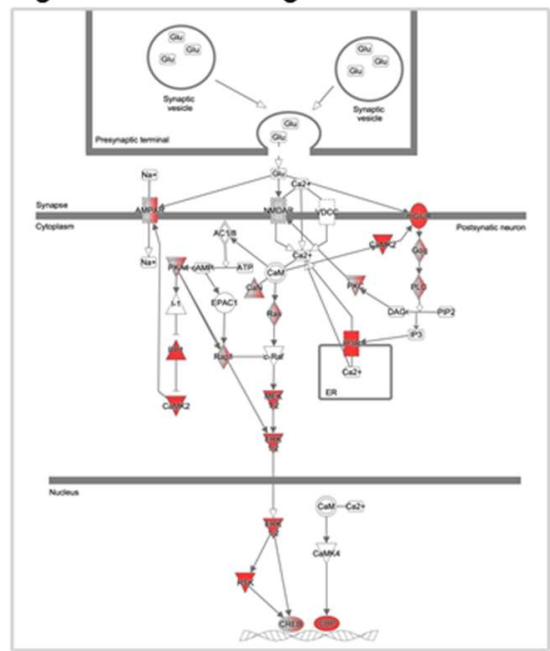

Aged GSK' 872 vs Aged Veh

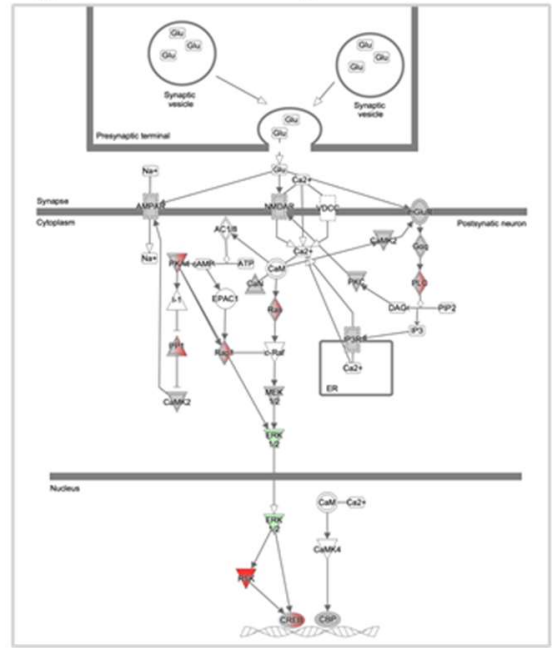

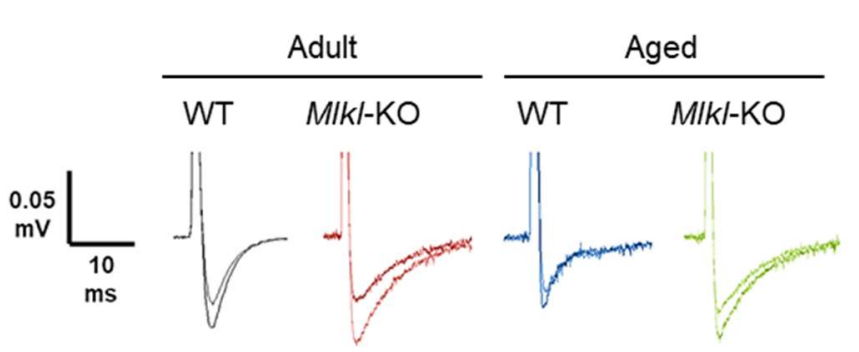
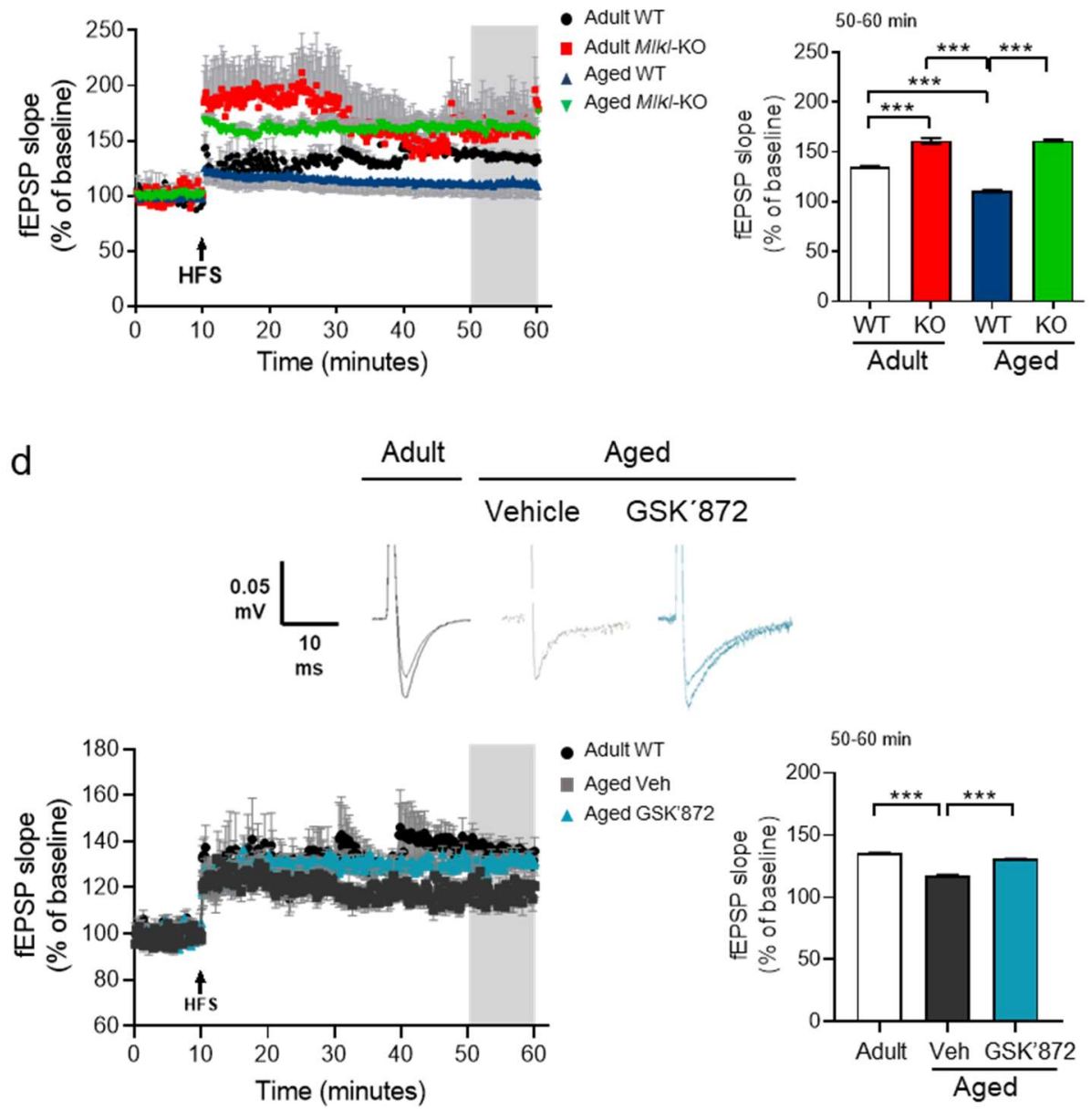

- Adult WT

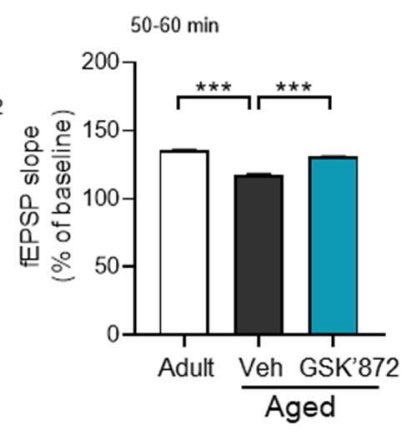


Figure 8. Increased synaptic long-term potentiation in the necroptosis-inhibited aging process. Schematic illustrating the canonical pathway annotation of "Synaptic long-term potentiation signaling". Top canonical pathway annotations were defined by ranking of absolute z-score between "normal" aging, MIkl-KO and GSK'872 analyses. Intensity of colour represents magnitude of change; red corresponds to upregulation compared to control within each respective analysis, while green represents downregulation. Molecules in grey were identified to be necroptosis-correlative alterations present within input dataset, but fell below the $20 \%$ cut-off, while molecules in white were not present within input dataset (See Supplementary Figures S8, S9) but changed less than $20 \%$ in analysis. Solid connecting lines represent a direct interaction, while dashed connecting lines indicate an indirect interaction. (b) Heat map of individual proteins assigned to the canonical pathway "Synaptic longterm potentiation signaling". Changes between normal aging and MIKl-KO or GSK'872 treated mice were expressed as fold change. (c) Canonical pathway annotation "Synaptic long-term potentiation signaling". Synaptic plasticity was evaluated by LTP magnitude measurement, generated by highfrequency stimulation (HFS) in the hippocampal CA1 area of WT and MIkl-KO mice from adult and aged groups. Average fEPSP slope was plotted considering the last 10 minutes of the recording. (d) "Synaptic LTP signaling" in aged mice treated with GSK'872 vs vehicle. LTP was generated by HFS in the CA1-CA3 hippocampal transmission and registered for $1 \mathrm{~h}$. The last 10 minutes were plotted as average fEPSP slope to evaluate statistical differences. Values are the result of the analysis of $\mathrm{N}=4$ mice per group ( $n=8-10$ slices per mice). Error bars, mean $\pm S E M,{ }^{* * *} p<0.005$. Statistical significance was determined by one-way ANOVA. 
bioRxiv preprint doi: https://doi.org/10.1101/2021.11.10.468052; this version posted November 11, 2021. The copyright holder for this preprint (which was not certified by peer review) is the author/funder. All rights reserved. No reuse allowed without permission.

Fig. 9

a Aged vs Adult
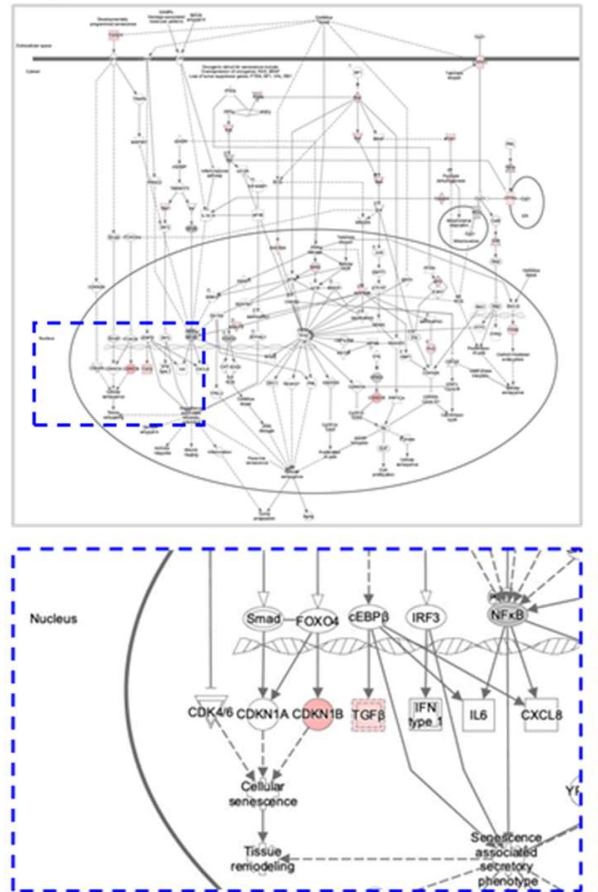

b collat

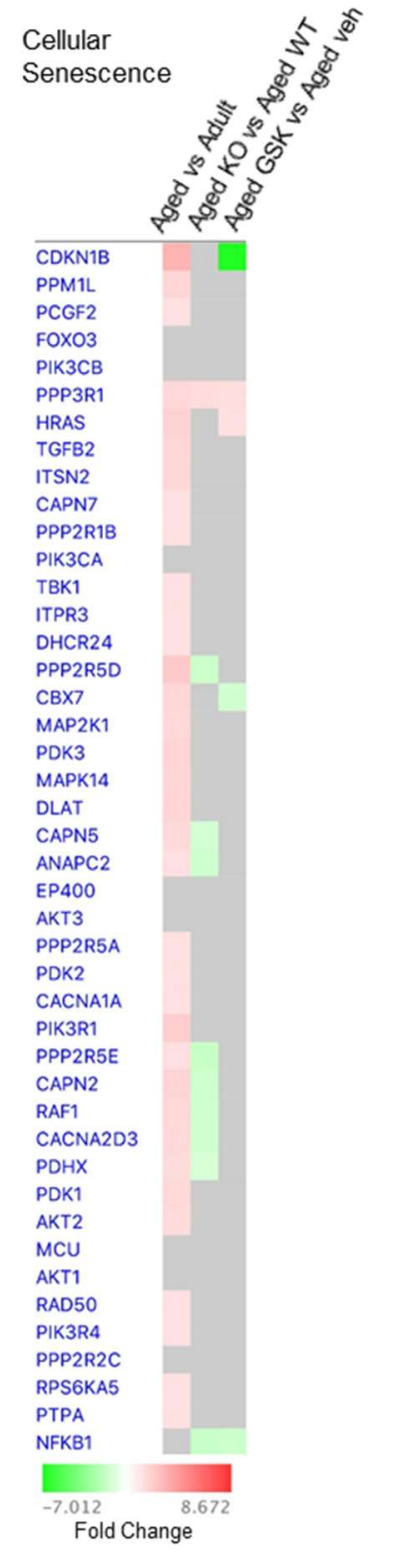

e
Aged MIkl-KO vs Aged WT
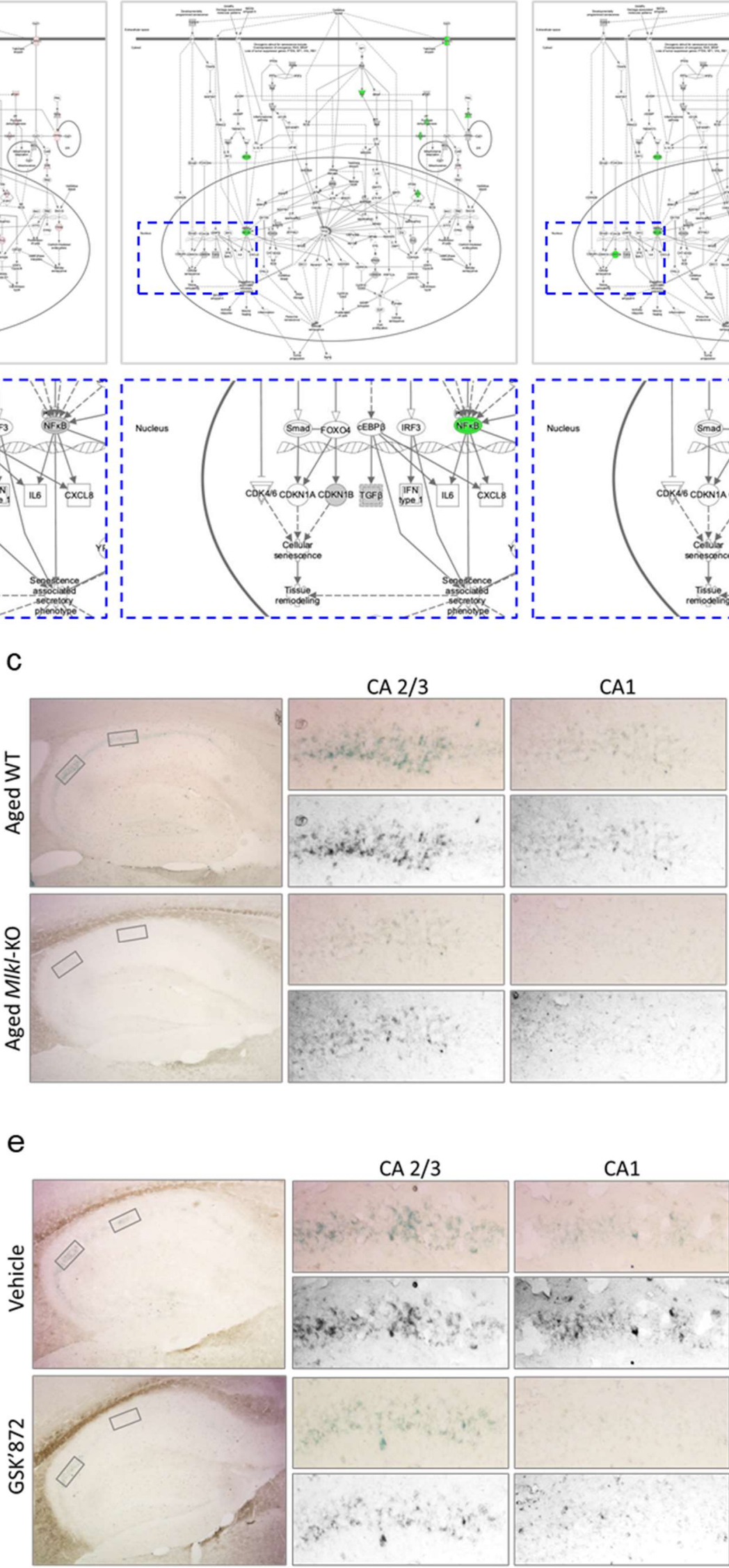

Aged GSK'872 vs Aged Veh

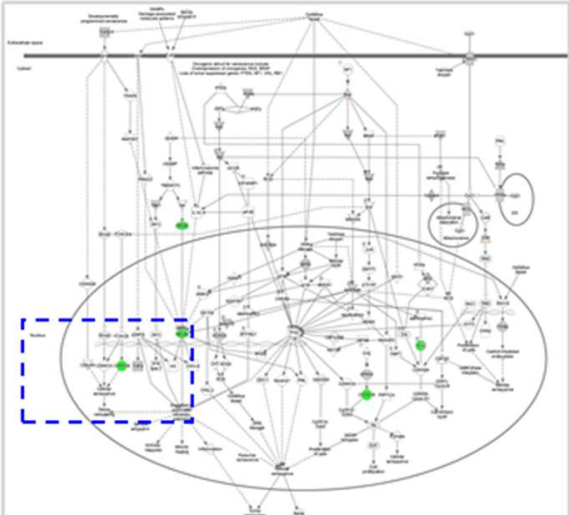

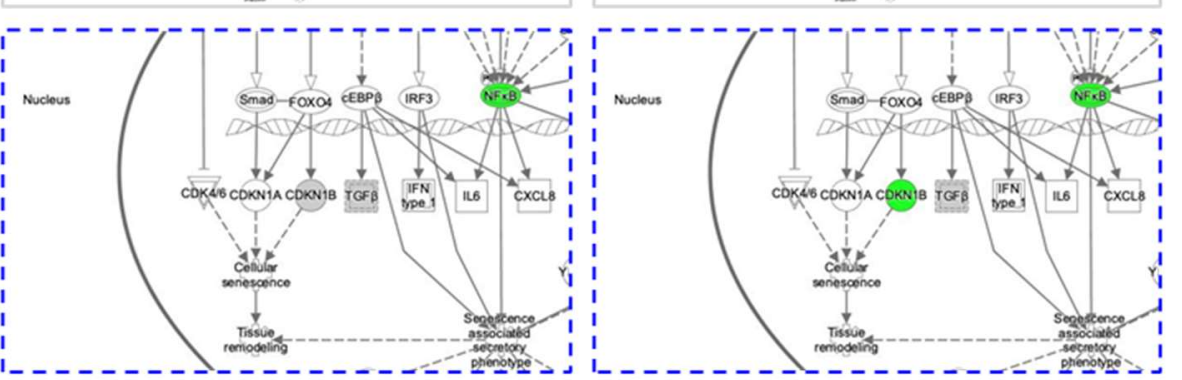

d

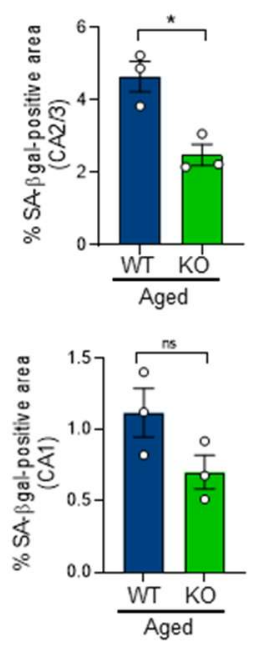

f
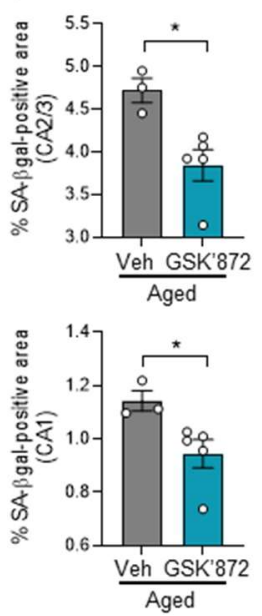
Figure 9. Decreased senescent phenotype in the hippocampus of aged mice with inhibited necroptosis (a) Schematic illustrating a top canonical pathway annotation as defined by ranking of absolute z-score between "normal" aging, MIkl-KO and GSK'872 analyses of "Cellular senescence signaling." Intensity of colour represents magnitude of change; red represents upregulation compared to control within each respective analysis, while green represents downregulation. Molecules in grey were identified to be necroptosis-correlative alterations present within input dataset, but fell below the $20 \%$ cut-off, while molecules in white were not present within input dataset (see Fig S8, S9). Magnifications of the global canonical pathway (blue dotted rectangle) show target molecules involved in cellular senescence execution in every condition (b) Heat map of individual proteins assigned to canonical pathway annotation of "cellular senescence". Changes between normal aging and MIkl-KO or GSK'872 treated mice were expressed as fold change. (c) Cellular senescence was measured as SA- $\beta$ galactosidase (SA- $\beta$ gal) activity and quantified in the hippocampal regions CA1 and CA2/3 of aged WT vs aged MIkl-KO mice. Magnifications of each hippocampal subfield shows SA- $\beta$ gal staining in grayscale. (d) Graphs show the percentage of the SA- $\beta$ gal-positive area in each brain region. Bars correspond to the mean $\pm S E M$ from $n=3$. (e) $S A-\beta$ gal activity in the $C A 1$ and $C A 2 / 3$ area of the hippocampus of aged-vehicle vs aged GSK' 872 treated mice. (f) Bars represent the mean \pm SEM from $n=3$. Significant differences were determined by unpaired T-test Student, ${ }^{*} p<0.05$. 